\title{
NUCLEOSYNTHESIS PREDICTIONS FOR INTERMEDIATE-MASS ASYMPTOTIC GIANT BRANCH STARS: COMPARISON TO OBSERVATIONS OF TYPE I PLANETARY NEBULAE*
}

\author{
Amanda I. Karakas ${ }^{1,5,6}$, Mark A. van Raai ${ }^{2}$, Maria Lugaro $^{2,7}$, N. C. Sterling ${ }^{3}$, And Harriet L. Dinerstein ${ }^{4}$ \\ ${ }^{1}$ Research School of Astronomy and Astrophysics, Mt. Stromlo Observatory, Weston Creek ACT 2611, Australia; akarakas@mso.anu.edu.au \\ ${ }^{2}$ Sterrenkundig Instituut, University of Utrecht, Postbus 80000, 3508 TA Utrecht, The Netherlands; M.A.vanRaai@students.uu.nl, m.a.lugaro@uu.nl \\ ${ }^{3}$ NASA Postdoctoral Program Fellow, Goddard Space Flight Center, Code 662, Greenbelt, MD 20771, USA; Nicholas.C.Sterling@nasa.gov \\ ${ }^{4}$ Department of Astronomy, University of Texas, 1 University Station, C1400, Austin, TX 78712-0259, USA; harriet@astro.as.utexas.edu \\ Received 2008 July 9; accepted 2008 September 3; published 2008 December 2
}

\begin{abstract}
Type I planetary nebulae $(\mathrm{PNe})$ have high $\mathrm{He} / \mathrm{H}$ and $\mathrm{N} / \mathrm{O}$ ratios and are thought to be descendants of stars with initial masses of $\sim 3-8 M_{\odot}$. These characteristics indicate that the progenitor stars experienced proton-capture nucleosynthesis at the base of the convective envelope, in addition to the slow neutron capture process operating in the He-shell (the s-process). We compare the predicted abundances of elements up to Sr from models of intermediate-mass asymptotic giant branch (AGB) stars to measured abundances in Type I PNe. In particular, we compare predictions and observations for the light trans-iron elements $\mathrm{Se}$ and $\mathrm{Kr}$, in order to constrain convective mixing and the $s$-process in these stars. A partial mixing zone is included in selected models to explore the effect of a ${ }^{13} \mathrm{C}$ pocket on the $s$-process yields. The solar-metallicity models produce enrichments of $[(\mathrm{Se}, \mathrm{Kr}) / \mathrm{Fe}] \lesssim 0.6$, consistent with Galactic Type I PNe where the observed enhancements are typically $\lesssim 0.3$ dex, while lower metallicity models predict larger enrichments of $\mathrm{C}, \mathrm{N}, \mathrm{Se}$, and $\mathrm{Kr}$. O destruction occurs in the most massive models but it is not efficient enough to account for the $\gtrsim 0.3$ dex O depletions observed in some Type I PNe. It is not possible to reach firm conclusions regarding the neutron source operating in massive AGB stars from $\mathrm{Se}$ and $\mathrm{Kr}$ abundances in Type I PNe; abundances for more $s$-process elements may help to distinguish between the two neutron sources. We predict that only the most massive $\left(M \gtrsim 5 M_{\odot}\right)$ models would evolve into Type I PNe, indicating that extra-mixing processes are active in lower-mass stars (3-4 $\left.M_{\odot}\right)$, if these stars are to evolve into Type I PNe.
\end{abstract}

Key words: nuclear reactions, nucleosynthesis, abundances - planetary nebulae: general - stars: AGB and post-AGB

Online-only material: color figures

\section{INTRODUCTION}

After the thermally-pulsing asymptotic giant branch (TP-AGB) phase is terminated, low-to-intermediate-mass stars $\left(\sim 0.8\right.$ to $\left.8 M_{\odot}\right)$ evolve to become post-AGB stars and possibly planetary nebulae (PNe), if the ejected envelopes have sufficient time to become ionized by the hot central stars before dissipating into the interstellar medium (ISM). For recent reviews of TP-AGB and post-AGB stars, see Herwig (2005) and van Winckel (2003), respectively. The illuminated PN is comprised of material from the deep convective envelope; hence, nebular abundances should reveal information about the efficiency of mixing events and chemical processing that took place during previous evolutionary phases, in addition to the initial composition of the parent star (Dopita et al. 1997; Karakas \& Lattanzio 2003a).

Briefly, during the TP-AGB phase, the He-burning shell becomes thermally unstable every $10^{4}$ years or so, depending on the core mass. The energy from the thermal pulse (TP) drives a convective zone in the He-rich intershell, which mixes the products of He-nucleosynthesis within this region. The energy provided by the TP expands the whole star, pushing the

\footnotetext{
* This paper includes data taken at The McDonald Observatory of The University of Texas at Austin.

5 Physics Division, Argonne National Laboratory, Argonne, IL 60439-4843.

6 Department of Astronomy and Astrophysics, University of Chicago, 5640 S. Ellis Avenue, Chicago, Illinois 60637.

7 Centre for Stellar \& Planetary Astrophysics, Monash University, Clayton VIC 3800, Australia.
}

$\mathrm{H}$-shell out to cooler regions where it is almost extinguished, and subsequently allowing the convective envelope to move inwards (in mass) to regions previously mixed by the flashdriven convective zone. This inward movement of the convective envelope is known as the third dredge-up (TDU), and is responsible for enriching the surface in ${ }^{12} \mathrm{C}$ and other products of He-burning, as well as heavy elements produced by the $s$-process. Following the TDU, the star contracts and the $\mathrm{H}$-shell is reignited, providing most of the surface luminosity for the next interpulse period. In intermediate-mass AGB stars with initial masses $\gtrsim 4 M_{\odot}$, the base of the convective envelope can dip into the top of the H-burning shell, causing proton-capture nucleosynthesis to occur there (hot bottom burning, HBB; see Lattanzio et al. 1996 for a review).

Abundances can be obtained from PN spectra for a number of elements including $\mathrm{C}, \mathrm{N}, \mathrm{O}, \mathrm{S}, \mathrm{Cl}$ and the noble gases $\mathrm{He}$, $\mathrm{Ne}$, and Ar (Aller \& Czyzak 1983; Kingsburgh \& Barlow 1994; Dopita et al. 1997; Stanghellini et al. 2000; Leisy \& Dennefeld 2006), and heavy elements synthesized by the $s$-process, including $\mathrm{Ge}, \mathrm{Se}, \mathrm{Kr}, \mathrm{Xe}, \mathrm{Ba}$, and $\mathrm{Br}$ (Pequignot \& Baluteau 1994; Dinerstein 2001; Sterling et al. 2002, 2007; Sharpee et al. 2007; Sterling \& Dinerstein 2008, hereafter SD08). Whereas the metallicity of a star is usually taken from the abundance of iron, for ionized nebulae, oxygen is generally the primary index of metallicity. However, $\mathrm{O}$ is not an ideal metallicity index for PNe, because it can be destroyed by $\mathrm{CNO}$ cycling and/or synthesized during helium burning. Consequently, Ar is sometimes used in place of O (Leisy \& Dennefeld 2006, SD08), under the assumption that it remains 
unchanged by AGB nucleosynthesis. Zinc is a better indicator of iron-group element abundances in nebulae than Fe itself, because $\mathrm{Zn}$ does not condense into dust as easily as Fe (York \& Jura 1982; Sembach et al. 1995; Welty et al. 1999) and can be observed in PNe (Dinerstein \& Geballe 2001; Dinerstein 2004). However, $\mathrm{Zn}$ is at the beginning of the $s$-process chain and might be produced to some extent in AGB stars. In order to interpret the elemental abundances in $\mathrm{PNe}$, it is important to determine which elements are produced, destroyed or left unaltered by AGB nucleosynthesis.

The set of elements heavier than Fe that can be detected in PNe include all even-numbered elements from $Z=30-36$, which lie at the light-element end of the $s$-process distribution, just below the first $s$-process peak at $\mathrm{Sr}, \mathrm{Y}$, and $\mathrm{Zr}(Z=38-40)$. In Karakas et al. (2007), we attempted to use Ge abundances derived from a small sample of PNe (Sterling et al. 2002) to try and constrain the amount of TDU mixing after the final few TPs. There, a hint was found that efficient TDU at the tip of the AGB may be required in order to match the abundances; however, stronger conclusions were precluded by model uncertainties, the small PNe sample size, and the large uncertainties of the Ge abundance determinations. In the present study, we proceed to analyze Se and $\mathrm{Kr}$ abundances from a much larger sample of 120 Galactic PNe from SD08, where the $s$-process enrichments were investigated for correlations with PN morphology and other nebular and stellar characteristics, as outlined in detail in Section 2. We also use $\mathrm{PNe}$ abundances of $\mathrm{C}, \mathrm{N}$, and $\mathrm{O}$ along with the abundances of post-AGB stars for comparison to the stellar models.

The composition of Type I PNe provides important constraints on nucleosynthesis and mixing in intermediate-mass AGB stars. These PNe tend to exhibit bipolar morphologies and have high $\mathrm{He} / \mathrm{H}$ and $\mathrm{N} / \mathrm{O}$ ratios characteristic of proton-capture nucleosynthesis (Stanghellini et al. 2006), suggesting that they are descendants of AGB stars with initial masses $\sim 3-8 M_{\odot}$ (Peimbert 1978; Kingsburgh \& Barlow 1994; Stanghellini et al. 2006). Low-mass AGB stars, by contrast, are defined as having initial masses $\sim 1-3 M_{\odot}$ and tend to show carbon and $s$-process element enrichments (Busso et al. 2001; Travaglio et al. 2004). In the Milky Way, the spatial distribution and kinematics of Type I PNe indicate that they are a young population (e.g., Corradi \& Schwarz 1995). This supports their identification with intermediate-mass stars, which have relatively short evolutionary lifetimes. In the Galaxy, intermediate-mass AGB stars are difficult to identify owing to a lack of reliable distances. This has resulted in a paucity of observational evidence for constraining stellar models, and especially the efficiency of the TDU in intermediate-mass AGB stars, which is still not well determined (Karakas et al. 2002; Ventura et al. 2002; Herwig 2004; Stancliffe et al. 2004). García-Hernández et al. (2006) identified several Galactic intermediate-mass AGB stars within a sample of $\mathrm{OH} / \mathrm{IR}$ stars (i.e., bright O-rich giants with large infrared (IR) excesses). The large enhancements of $\mathrm{Rb}$ found by these authors, combined with the fact that these stars are O-rich, support the prediction that HBB and an efficient TDU has occurred in these stars. At lower metallicities, constraints are provided by observations of luminous O-rich AGB stars in the Large and Small Magellanic Clouds (LMC and SMC, respectively) that are rich in Li and $s$-process elements (Wood et al. 1983; Smith \& Lambert 1989; Plez et al. 1993).

In Section 2, we begin with a summary of observational results for $n$-capture elements in PNe. We review the numerical method and present the stellar models in Section 3, and outline the results in Section 4. In Section 5, we discuss our findings and their implications, and in Section 6 we summarize our conclusions.

\section{OBSERVATIONAL CONSTRAINTS}

The low cosmic abundances of $n$-capture elements cause their spectroscopic features to be very weak. For that reason, transiron elements were not identified in the spectrum of a PN until 1994 (Pequignot \& Baluteau 1994). However, in the last few years, there has been considerable progress in the detection of emission lines from and derivation of abundances for transiron elements in PNe (e.g., Dinerstein \& Geballe 2001; Sterling et al. 2007; Sharpee et al. 2007, SD08).

The most comprehensive study is that of SD08, who conducted the first large-scale survey of $n$-capture elements in PNe. They determined $\mathrm{Kr}$ and Se abundances in $120 \mathrm{PNe}$ from the near-infrared (NIR) emission lines [Kr III] $2.199 \mu \mathrm{m}$ and [Se IV] $2.287 \mu \mathrm{m}$ identified by Dinerstein (2001), and corrected for the abundances of unobserved Se and $\mathrm{Kr}$ ions using analytical formulae derived from a grid of photoionization models (Sterling et al. 2007). This survey increased the number of PNe with determined $n$-capture element abundances by nearly an order of magnitude, enabling a detailed study of $s$-process enrichments in $\mathrm{PNe}$ as a population as well as an assessment of enrichment patterns in different classes of PNe. In order to normalize the abundances of the $s$-process products to the initial metallicity of each PN, the Se and $\mathrm{Kr}$ abundances were compared to those of $\mathrm{O}$ and $\mathrm{Ar}$ reported in the literature. Oxygen was utilized as a reference element for PNe with low-mass progenitors, since its abundance is generally the most reliably determined in PNe, while Ar was used for objects with higher-mass central stars that may have experienced $\mathrm{O}$ destruction during HBB. Overall, 41 of the $94 \mathrm{PNe}$ with derived Se and/or $\mathrm{Kr}$ abundances or meaningful upper limits were found to be enriched in these elements, with the average $[\mathrm{Se} /(\mathrm{O}, \mathrm{Ar})]=0.31$ and $[\mathrm{Kr} /(\mathrm{O}, \mathrm{Ar})]=$ $0.98 .{ }^{8} \mathrm{SD} 08$ interpreted these enrichments as evidence for in situ $s$-process nucleosynthesis and dredge-up in PN progenitor stars.

SD08 observed significant numbers of PNe with intermediatemass progenitor stars, as evidenced by their Type I compositions and/or bipolar morphologies (29 and 28 objects, respectively; see their Table 15). They found that Type I and bipolar PNe exhibit smaller Se and $\mathrm{Kr}$ enrichments on average than other PNe. In Figure 1, we display $[\mathrm{Se} /(\mathrm{O}, \mathrm{Ar})]$ and $[\mathrm{Kr} /(\mathrm{O}, \mathrm{Ar})]$, separated into $0.1 \mathrm{dex}$ bins, for non-Type I, Type I, and bipolar PNe. The distribution of $\mathrm{Se}$ and $\mathrm{Kr}$ enrichments is clearly skewed to smaller values for Type I PNe than other objects, and to a lesser extent the same is true of bipolar PNe. Notably, all of the Type I PNe observed by SD08 exhibit modest (less than a factor of 2 , or 0.3 dex) or no Se and $\mathrm{Kr}$ enrichments. These values are significantly lower than the average values for non-Type I PNe: 0.36 dex for [Se/ $(\mathrm{O}, \mathrm{Ar})]$ and $1.02 \mathrm{dex}$ for $[\mathrm{Kr} /(\mathrm{O}, \mathrm{Ar})]$. In contrast, while some bipolar PNe are among the objects with the lowest $[\mathrm{Se} /(\mathrm{O}, \mathrm{Ar})]$ and $[\mathrm{Kr} /(\mathrm{O}, \mathrm{Ar})]$, others display enrichments approaching a factor of 10 .

Figure 2 shows $[\mathrm{Se} /(\mathrm{O}, \mathrm{Ar})]$ and $[\mathrm{Kr} /(\mathrm{O}, \mathrm{Ar})]$ as a function of the logarithmic $\mathrm{He} / \mathrm{H}, \mathrm{N} / \mathrm{O}$, and $\mathrm{C} / \mathrm{O}$ abundances of $\mathrm{PNe}$ from the SD08 sample, including upper limits (open symbols) for objects without detected $\mathrm{Se}$ and/or Kr emission. This figure

\footnotetext{
8 We use the notation $[\mathrm{Se} /(\mathrm{O}, \mathrm{Ar})]$ and $[\mathrm{Kr} /(\mathrm{O}, \mathrm{Ar})]$ of SD08 to emphasize the use of different reference elements for $\mathrm{PNe}$ with different progenitor masses, where $[\mathrm{X} / \mathrm{Y}]=\log _{10}(\mathrm{X} / \mathrm{Y})-\log _{10}(\mathrm{X} / \mathrm{Y})_{\odot}$.
} 
not only illustrates the lower average $\mathrm{Se}$ and $\mathrm{Kr}$ abundances of Type I PNe relative to other objects, but also that upper limits to the Se and $\mathrm{Kr}$ abundances in many Type I PNe allow for at most marginal $s$-process enrichments. SD08 found that $s$-process enrichments are positively correlated with $\mathrm{C} / \mathrm{O}$ in non-Type I PNe (bottom panels of Figure 2), a result that agrees with studies of AGB (Smith \& Lambert 1990; Abia et al. 2002) and post-AGB stars (van Winckel 2003). Type I PNe often exhibit low $\mathrm{C} / \mathrm{O}$ ratios $(<1)$, in agreement with predictions from intermediate-mass stars with HBB. HBB converts dredgedup ${ }^{12} \mathrm{C}$ into ${ }^{14} \mathrm{~N}$, thereby preventing or delaying the formation of C-rich AGB stars (Frost et al. 1998). However, some bipolar $\mathrm{PNe}$ display significant enrichments of both $\mathrm{C}$ and $s$-process nuclei, as well as non-Type I compositions. These objects may be descendants of low-mass binary star systems in which the bipolar morphology is a result of binary interactions (Soker 1997; Balick \& Frank 2002). This suggests that some bipolar $\mathrm{PNe}$ are not descendants of intermediate-mass stars, and that morphology is not as reliable an indicator of progenitor mass as is chemical composition.

Sharpee et al. (2007) derived $n$-capture element abundances in five PNe from high-resolution optical spectra. They identified emission lines of several $n$-capture elements, including $\mathrm{Br}, \mathrm{Kr}$, $\mathrm{Xe}, \mathrm{Rb}, \mathrm{Ba}$, and $\mathrm{Pb}$ in each of these objects, and derived abundances for $\mathrm{Br}, \mathrm{Kr}$, and $\mathrm{Xe}$. They found that $\mathrm{Kr}$ and $\mathrm{Xe}$ are enriched in three of the five observed PNe, by $0.3-0.9$ dex. The two objects in their sample that do not exhibit $s$-process enrichments, NGC 2440 and IC 2501, are both Type I PNe according to the classification scheme of Peimbert (1978). The $[\mathrm{Kr} / \mathrm{Ar}]$ and $[\mathrm{Xe} / \mathrm{Ar}]$ values of these two objects are consistent within the uncertainties with the solar values, except for Xe in NGC 2440, which may be mildly subsolar. These results for [Kr/Ar] are consistent with the findings of SD08 for their larger sample of Type I PNe.

The NIR [Kr III] and [Se IV] lines utilized by SD08 have also been detected in two PNe belonging to metal-poor Milky Way dwarf satellite galaxies (Wood et al. 2006, H. L. Dinerstein et al. 2008 , in preparation). Such observations can provide important constraints on AGB models and nucleosynthetic yields for subsolar metallicities. One of the observed objects, LMC SMP 62, is $\mathrm{N}$-rich $(\mathrm{N} / \mathrm{O} \sim 0.5)$ and, hence, considered a Type I PN in the context of the LMC, where the threshold value of $\mathrm{N} / \mathrm{O}$ for Type I objects is taken to be lower than for Galactic PNe (Dopita \& Meatheringham 1991; Leisy \& Dennefeld 2006). While the [ $\mathrm{Kr}$ III] line is not detected (and does not provide a useful abundance limit), the [Se IV] line yields an Se abundance $\sim 15 \%$ solar (H. L. Dinerstein et al. 2008, in preparation). Not only is Se not enriched, it is actually deficient relative to the $\alpha$-elements; the abundances of $\mathrm{O}, \mathrm{Ne}, \mathrm{S}$, and $\mathrm{Ar}$ in SMP 62 are $\sim 30-40 \%$ solar (Leisy \& Dennefeld 2006; BernardSalas et al. 2008). ${ }^{9}$ This is reminiscent of the abundance pattern in LMC red giants, where light $s$-process elements, such as Y and $\mathrm{Zr}$, are markedly deficient relative to iron and some $\alpha$ elements (Pompeia et al. 2006; Mucciarelli et al. 2008). In contrast, Hen 2-436, a PN in the Sagittarius dSph galaxy, displays Se and $\mathrm{Kr}$ abundances as high as those for some of the most enriched Galactic objects shown in Figure 1. However, Hen 2-436 is

\footnotetext{
9 Using Spitzer spectra, Bernard-Salas et al. (2008) found higher $\mathrm{Ne} / \mathrm{H}$ than Leisy \& Dennefeld (2006). However, they adopted a higher value for the solar $\mathrm{Ne}$ abundance, and, consequently, their ratio to solar was similar. They also derived a low value for $\mathrm{S} / \mathrm{H}$, but the significance of this result is unclear, since these authors also found that Galactic PNe had subsolar sulfur abundances (Pottasch \& Bernard-Salas 2006).
}

Table 1

Details of the Stellar Models

\begin{tabular}{|c|c|c|c|c|c|c|c|c|c|c|c|}
\hline ass & $Z$ & Ps & $T_{1}$ & $T_{\text {bce }}^{\max }$ & $M_{\text {dred }}$ & $M_{\mathrm{env}}$ & $\mathrm{H}$ & & ${ }^{15} \mathrm{C}$ & $\mathrm{N} /$ & $\mathrm{He} /$ \\
\hline & 02 & 26 & 302 & & (6 & & & 1.27 & 108 & & 12 \\
\hline & & & & & & & & & & & \\
\hline & & & & & & & & & & & \\
\hline & & & & & & & & & & & \\
\hline & & & & & & & & & & & \\
\hline & & 4 & & & & & & & & & \\
\hline & & 21 & & & & & & & & & \\
\hline & & 51 & 36 & & & & & & & & \\
\hline & & 2 & & & & & & & & & 10 \\
\hline & & & & & & & & & & & \\
\hline & & 6 & 3 & & & & & & & & \\
\hline & & o1 & & 04. & -1 & $1.1+1$ & & 3.62 & 10.8 & & 0.15 \\
\hline
\end{tabular}

Notes. ${ }^{\text {a }}$ Computed with Reimers mass loss on the AGB. All other models have the Vassiliadis \& Wood (1993) mass loss.

C-rich rather than N-rich, and, therefore, is a non-Type I PN, with an estimated initial stellar mass of $\sim 1.2 M_{\odot}$ (Dudziak et al. 2000; Zijlstra et al. 2006).

Zinc is detectable in PNe via the [Zn Iv] $3.625 \mu \mathrm{m}$ finestructure line identified by Dinerstein \& Geballe (2001). This line has recently been observed in about a dozen PNe belonging to the Milky Way and its dwarf satellites (Dinerstein et al. 2007, H. L. Dinerstein et al. 2008, in preparation, hereafter DGS08). Two of these objects exhibit Type I compositions: LMC SMP 62, discussed above, and the Galactic PN M 1-40 (Górny et al. 2004). The gaseous $\mathrm{Zn}$ abundance in M 1-40 is $\sim 80 \%$ solar, which is consistent with its approximately solar $\mathrm{Ne}$ and S abundances. Even allowing for modest depletion into dust, which would raise the total $\mathrm{Zn}$ abundance by $0.1-0.2$ dex (assuming conditions typical of the warm ISM; see Section 1), it does not appear that $\mathrm{Zn}$ is significantly enriched in M 1-40. In LMC SMP 62, the measured $\mathrm{Zn}$ abundance is lower relative to solar than the abundances of $\alpha$ elements such as $\mathrm{O}, \mathrm{Ne}$, and $\mathrm{S}$ (Dinerstein et al. 2007) by a factor very similar to the deficiency in Se. In this context, it is interesting that several of the irongroup elements, including $\mathrm{Ni}$ and $\mathrm{Cu}$ (which are adjacent to $\mathrm{Zn}$ ), display behavior similar to light $n$-capture elements, which are also deficient relative to Fe in LMC giants (Pompeia et al. 2006; Mucciarelli et al. 2008).

\section{THE STELLAR MODELS}

The numerical method we use has been previously described in detail (Lugaro et al. 2004; Karakas et al. 2006). We first compute the stellar structure using the Mt. Stromlo Stellar Structure code (Lattanzio 1986), where each model is evolved from the zero-age main sequence to near the tip of the TP-AGB. For the models considered in this study, we do not evolve to the post-AGB phase due to convergence difficulties that occur when the envelope mass is reduced below $\sim 1.5 M_{\odot}$; see Karakas \& Lattanzio (2007) for more details. The structure is then used as input into a postprocessing nucleosynthesis code where we obtain abundances for many more species (up to 166) than are included in the structure model (6 species).

The stellar models we computed (see Table 1) cover a mass range of 3.0-6.5 $M_{\odot}$ and metallicities $0.2-1.0 Z_{\odot}$. The metallicities were chosen to reflect the composition of most of the PNe in the SD08 sample, where O and Ar abundances were used as metallicity indicators. We include a $2.5 M_{\odot}, Z=0.008$ model as an example of a low-mass, low-metallicity progenitor 

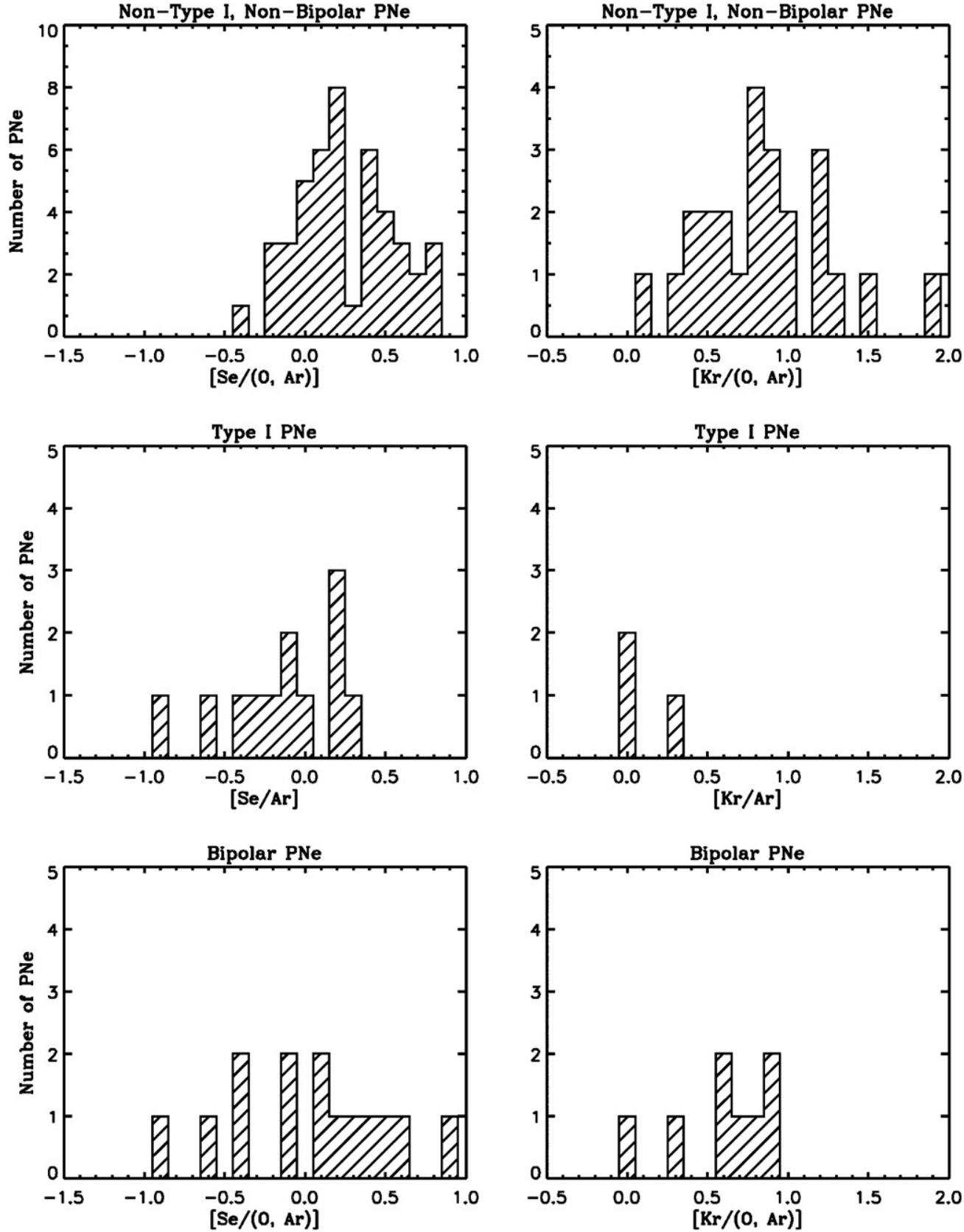

Figure 1. Histograms of Se and $\mathrm{Kr}$ abundances from SD08, separated into 0.1 dex bins, for nonbipolar, non-Type I PNe (top); Type I PNe (middle); and bipolar PNe (bottom). Data are shown only for objects in which Se and/or Kr emission was detected. Type I and (to a lesser extent) bipolar PNe exhibit smaller enrichments than PNe with less massive progenitor stars.

that would lead to a non-Type I PN composition. All models use scaled solar abundances from Anders \& Grevesse (1989) except for the $Z=0.012$ models, which are computed with the revised solar elemental abundances from Asplund et al. (2005) for comparison. The Reimers (1975) mass loss was used on the first giant branch, with the parameter $\eta=0.4$, and the Vassiliadis $\&$ Wood (1993) mass loss on the AGB. For comparison, we include results for a $5 M_{\odot}, Z=0.02$ model computed with Reimers mass loss on the AGB, with the parameter $\eta=3.5$.

In Table 1, we summarize structural information about the models. Most of the structure calculations are taken from Karakas \& Lattanzio (2007), with the $Z=0.012$ models previously discussed in Karakas et al. (2007). In Table 1, we present the initial mass and metallicity, $Z$; the total number of TPs computed; the maximum temperature in the He-shell,
$T_{\mathrm{He}}^{\mathrm{max}}$; the maximum temperature at the base of the convective envelope, $T_{\mathrm{bce}}^{\max }$; the total mass dredged into the envelope during the TP-AGB, $M_{\text {dred }}$; the final envelope mass $M_{\text {env }}$; and whether or not HBB occurs. In Table 1, masses are given in solar units, temperatures in millions of Kelvin, metallicity $Z$ is the fraction by mass of metals, and elemental and isotopic ratios are in terms of number ratios.

\subsection{Type I PNe and $H B B$}

Observations suggest that the Type I PN progenitor mass range is $\sim 3-8 M_{\odot}$ (e.g., Peimbert 1990; Stanghellini et al. 2006). For the purpose of this study, we consider models with masses between 3 and $6.5 M_{\odot}$, noting that more massive AGB stars would likely evolve too quickly to form observable 

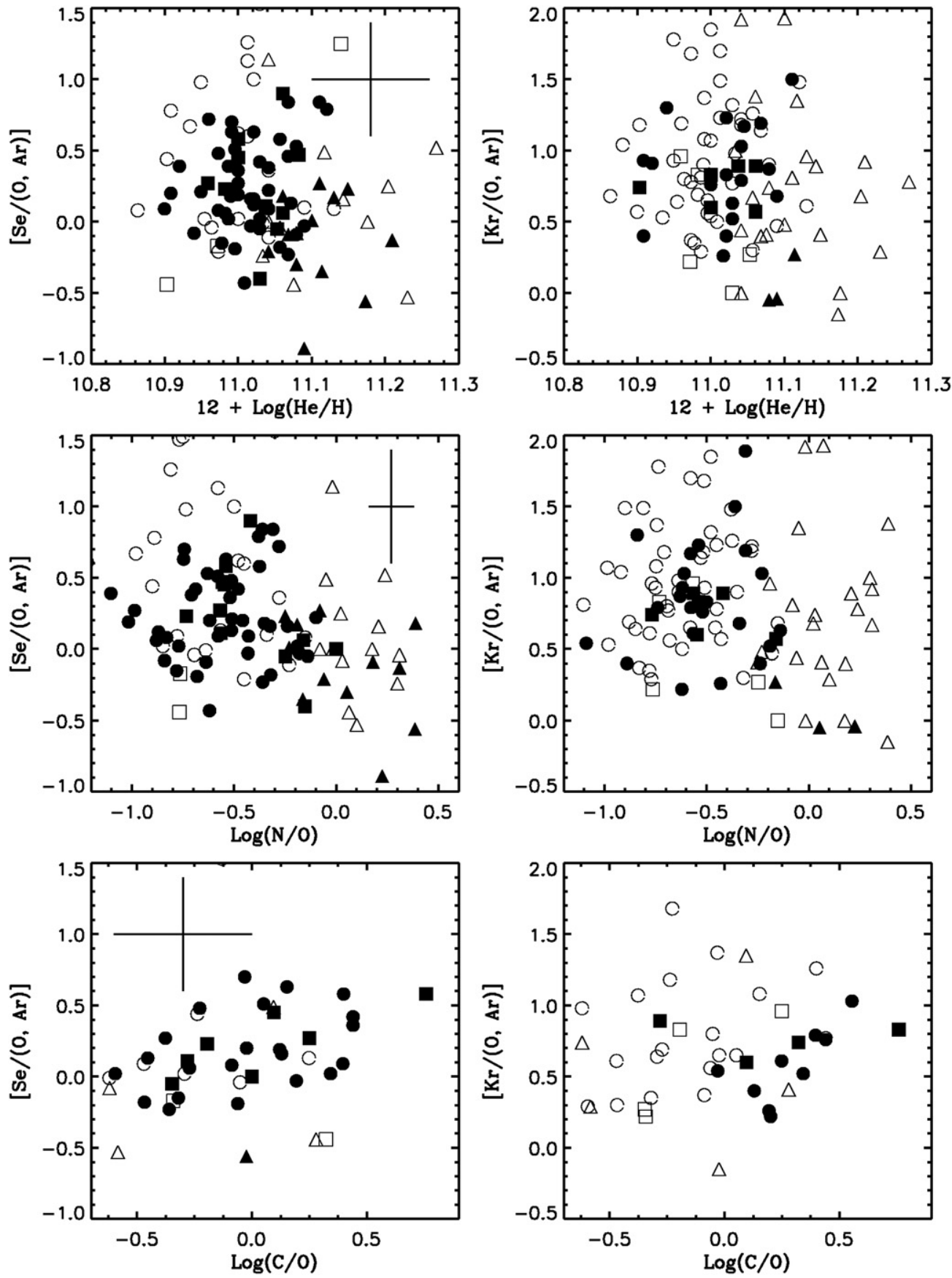

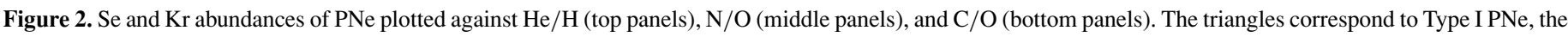

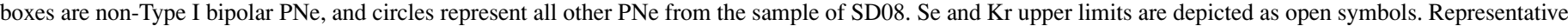

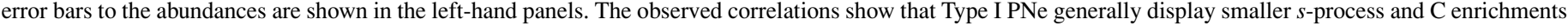

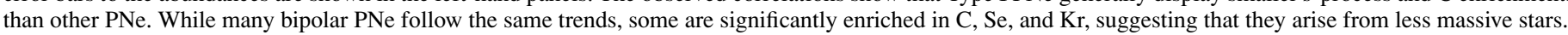

PNe. This mass range encompasses the full range of AGB nucleosynthetic behavior: (1) models that experience the TDU but not HBB, and (2) models that undergo the second dredge-up (SDU) and HBB in addition to the TDU. From inspection of Table 1 , the $2.5,3$, and $4 M_{\odot}$ models fall into the first category while the 5,6 , and $6.5 M_{\odot}$ models fall into the latter category. The more massive objects that experience both the SDU and $\mathrm{HBB}$ are often defined as intermediate-mass AGB stars (see Karakas et al. 2006; Herwig 2005).

Only models with HBB (i.e., models with initial masses $m \gtrsim 5 M_{\odot}$ ) would evolve into Type I PNe. Hence, efficient extra mixing between the base of the convective envelope and a region hot enough to allow some $\mathrm{CN}$ cycling presumably operates to produce the observed Type I nucleosynthethic signature of high $\mathrm{He} / \mathrm{H}$ and $\mathrm{N} / \mathrm{O}$ ratios in the lower-mass $\sim 3-4 M_{\odot}$ stars. The physical mechanism that could cause such efficient extra mixing is not known, although stellar rotation is one possible candidate (see below). It is probably not thermohaline mixing, which is only efficient in stars less massive than $2 M_{\odot}$ (Eggleton et al. 2006; Charbonnel \& Zahn 2007). The idea that extra-mixing processes are operating in low-mass carbon-rich AGB stars is supported by observational evidence, including lower than predicted ${ }^{12} \mathrm{C} /{ }^{13} \mathrm{C}$ ratios observed in N-type AGB stars (Abia \& Isern 1997). The $\mathrm{C}, \mathrm{O}$, and $\mathrm{Al}$ isotopic ratios found in meteoritic 
silicon carbide $(\mathrm{SiC})$ and oxide grains from AGB stars also point toward some sort of extra-mixing process operating in the parent stars (Busso et al. 1999; Nollett et al. 2003; Zinner et al. 2006; Zinner 2008). In these cases, the mixing should not be so efficient to prevent the formation of a carbon-rich atmosphere.

Some fraction of Type I and bipolar PNe presumably formed from low-mass stars via binary evolution (Soker 1997; Balick \& Frank 2002; Moe \& De Marco 2006), although it is unclear how this would lead to the high $\mathrm{He} / \mathrm{H}$ and N/O ratios observed. Twodimensional simulations of a star with a point-mass companion in a very close orbit suggested that close binary evolution has little effect on the deep interior structure, despite producing large distortions to the outer layers (Deupree \& Karakas 2005). However, these simulations did not go beyond the point where the stars begin to fill their Roche lobes. Rapid stellar rotation, in combination with magnetic fields, has also been proposed as a mechanism to shape bipolar $\mathrm{PNe}$ and to produce the overabundances of $\mathrm{He}$ and $\mathrm{N}$ in single intermediate-mass progenitors $\left(M \gtrsim 3 M_{\odot}\right)$ (Calvet \& Peimbert 1983; Gorny et al. 1997; García-Segura et al. 1999; Dobrinčić et al. 2008). Further modeling is required to address how effectively binary interactions, rotation, and/or magnetic fields can affect the internal structure and nucleosynthesis of AGB stars.

In Figure 3, we plot the temporal evolution of the core, and the temperature in the He-shell and at bottom of the convective envelope for the $6.5 M_{\odot}, Z=0.012$ model. The mass of the $\mathrm{H}$-exhausted core shows evidence of efficient TDU whereas the He-shell temperature shows that the majority of the TPs reach peak temperatures greater than $300 \times 10^{6} \mathrm{~K}$, the temperature at which the ${ }^{22} \mathrm{Ne}$ neutron source is activated. HBB prevented several models in Table 1 from becoming carbon rich, and also resulted in low ${ }^{12} \mathrm{C} /{ }^{13} \mathrm{C}$ ratios that approach the equilibrium ratio of $\sim 4$. The $\mathrm{He} / \mathrm{H}$ and $\mathrm{N} / \mathrm{O}$ ratios are also higher for models with HBB compared to the lower-mass models. HBB is more efficient at lower metallicities for a given mass owing to the higher temperatures reached at the base of the convective envelope. For example, the $6.5 M_{\odot}, Z=0.012$ model (corresponding to the Asplund et al. (2005) solar abundances) displays a higher $\mathrm{N} / \mathrm{O}$ ratio than the $Z=0.02$ model of the same mass. The final $\mathrm{N}$ abundance is similar in each of these two models, despite a difference of a factor of 1.86 in the initial $\mathrm{N}$ abundances, showing that $\mathrm{CNO}$ processing is more efficient in the low-metallicity case.

\subsection{The Nuclear Network}

The most important addition to our models for this study is the extension of the nuclear network to include elements heavier than iron up to niobium (M. A. van Raai et al. 2008, in preparation). We have also made an update of our reaction-rate library - starting from the library described in Karakas et al. (2007), we have included neutron-capture cross-sections from the Bao et al. (2000) compilation. We include 166 species from protons to sulfur and iron to niobium, with a total of 1285 reaction rates corresponding to all $\beta$-decay, $p, \alpha$, and $n$-capture reactions on all species in the network. We also have two other networks that we use to obtain information for the $\mathrm{S}$ and Ar isotopes. The first has 125 species and includes all stable species from $\mathrm{H}$ to ${ }^{62} \mathrm{Ni}$, while the second has 156 species and includes all stable species from $\mathrm{H}$ to As (Karakas et al. 2007).

As done in Karakas et al. (2007), we use a "double neutronsink" description (Herwig et al. 2003) to account for neutron captures on species heavier than that at the end of the network
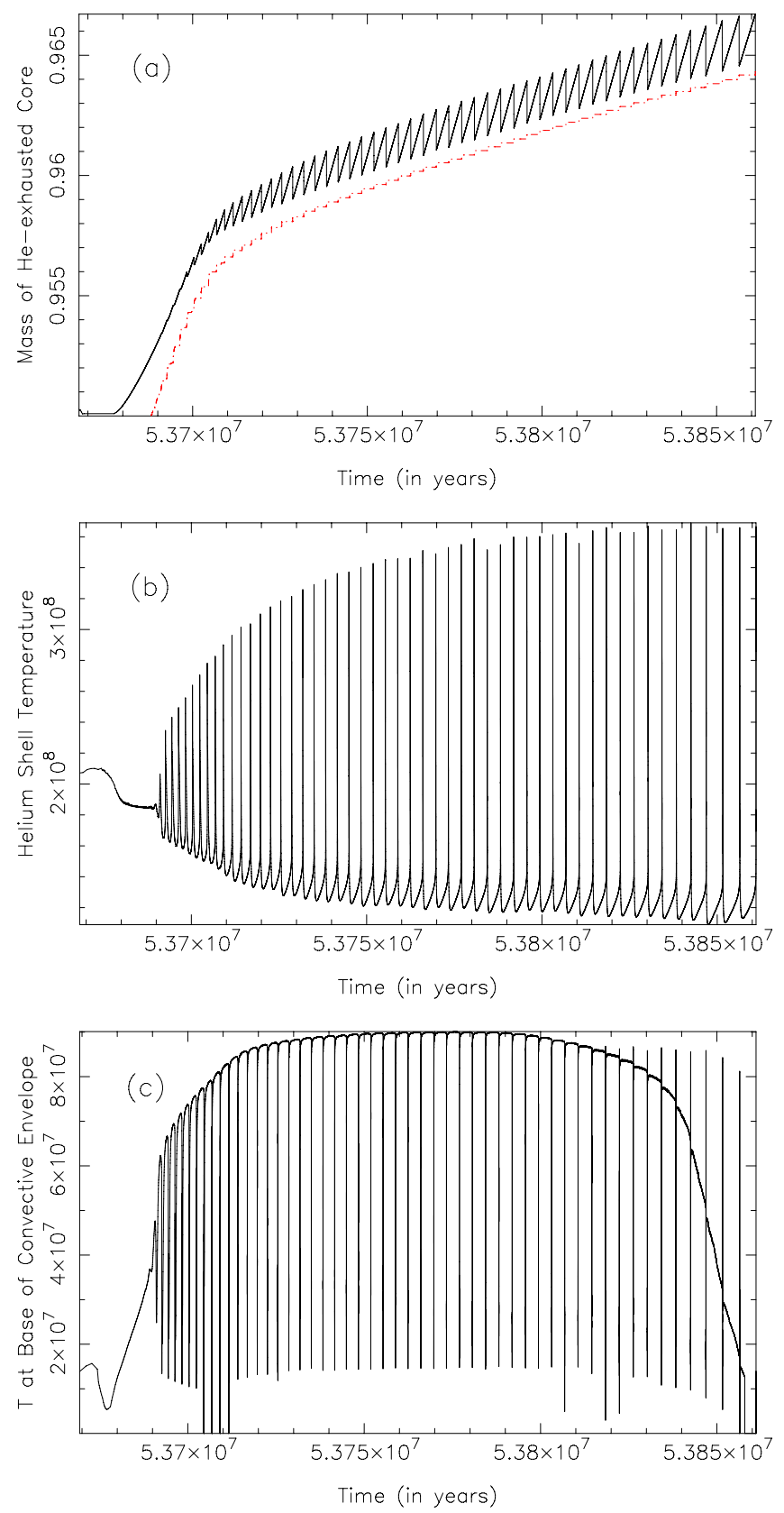

Figure 3. Temporal evolution of the (a) hydrogen (solid line) and heliumexhausted (dashed line) cores, (b) the He-shell temperature, and (c) the temperature at the base of the convective envelope for the $6.5 M_{\odot}, Z=0.012$ model during the TP-AGB.

(A color version of this figure is available in the online journal.)

$\left({ }^{97} \mathrm{Nb}\right.$ in this case). The two artificial species are linked by the reactions ${ }^{97} \mathrm{Nb}(n, \gamma){ }^{98} \mathrm{~g}$ and ${ }^{98} \mathrm{~g}(n, L){ }^{98} \mathrm{~g}$, where ${ }^{98} \mathrm{~g}$ has an initial abundance equal to the sum of the solar abundances from Mo to $\mathrm{Bi}$. The second artificial particle, $L$, is equivalent to the number of neutrons captured beyond $\mathrm{Nb}$. The ratio $\left(L /{ }^{98} \mathrm{~g}\right)$ is a description of the neutrons captured per seed nucleus and could, in principle, be related to the $s$-process distribution. Note that in the 156 and 166 networks, the species ${ }^{34} \mathrm{~S}$ is the sum of all species from ${ }^{34} \mathrm{~S}$ to $\mathrm{Mn}$, and the reaction ${ }^{34} \mathrm{~S}(n, \gamma){ }^{35} \mathrm{~S}$ is assigned an averaged cross-section value in order to represent all nuclei from ${ }^{34} \mathrm{~S}$ to $\mathrm{Mn}$. 


\subsection{Neutron Sources in Intermediate-Mass AGB Stars}

The $s$-process is driven by the production of free neutrons that are subsequently captured by Fe-peak seed nuclei to form heavier elements. The ${ }^{22} \mathrm{Ne}(\alpha, n)^{25} \mathrm{Mg}$ reaction was first suggested as a neutron source in stars by Cameron (1960). Later, Truran \& Iben (1977) and Cosner et al. (1980) suggested that it was the dominant source in intermediate-mass AGB stars, because the He-shells of these stars reached high enough temperatures $\left(T \gtrsim 300 \times 10^{6} \mathrm{~K}\right)$ to allow for this reaction to be efficiently activated (see the middle panel of Figure 3 as an example). These temperatures are reached only in the last few TPs of a lower-mass star. Truran \& Iben (1977) found that the ${ }^{22} \mathrm{Ne}$ source results in enhanced levels of the $n$-rich element $\mathrm{Rb}$ owing to the high neutron densities (up to $\sim 10^{13}$ neutrons $\mathrm{cm}^{-3}$ ), as well as increases in the heavy $\mathrm{Mg}$ isotopes ${ }^{25} \mathrm{Mg}$ and ${ }^{26} \mathrm{Mg}$ from the competing reactions ${ }^{22} \mathrm{Ne}(\alpha, n){ }^{25} \mathrm{Mg}$ and ${ }^{22} \mathrm{Ne}(\alpha, \gamma){ }^{26} \mathrm{Mg}$ (Kaeppeler et al. 1994; Karakas et al. 2006). Fenner et al. (2003) compared results from a chemical evolution model with observations of the neutron-rich $\mathrm{Mg}$ isotopes and concluded that an extra production site besides Type II supernovae was necessary, and that massive AGB stars are a good candidate for such a site. There is, however, some uncertainty at what Galactic epoch AGB stars started contributing to the chemical enrichment of the Galaxy (Simmerer et al. 2004; Meléndez \& Cohen 2007). It is also unknown to what extent intermediate-mass stars contribute to the Galactic inventory of $s$-process elements (e.g., Travaglio et al. 2004).

The other potential source of neutrons in AGB stars is the ${ }^{13} \mathrm{C}(\alpha, n){ }^{16} \mathrm{O}$ reaction, which operates at lower temperatures $\left(T \gtrsim 90 \times 10^{6}\right)$ than the ${ }^{22} \mathrm{Ne}$ source. Observational and theoretical evidence suggests this is the dominant neutron source in low-mass AGB stars (Smith et al. 1987; Gallino et al. 1998). To operate efficiently, this reaction requires more ${ }^{13} \mathrm{C}$ than is left over from $\mathrm{CN}$ cycling in the $\mathrm{H}$-shell; hence, some mechanism to mix protons from the H-rich envelope into the intershell is needed to produce the extra ${ }^{13} \mathrm{C}$. In our models, protons are mixed into the intershell region by artificially adding a partial mixing zone (PMZ) at the deepest extent of each TDU. These protons are quickly captured by the abundant ${ }^{12} \mathrm{C}$ to form ${ }^{13} \mathrm{C}$ and ${ }^{14} \mathrm{~N}$, resulting in the formation of a ${ }^{13} \mathrm{C}$ pocket. In the ${ }^{13} \mathrm{C}$ pocket, neutrons are liberated by the reaction ${ }^{13} \mathrm{C}(\alpha, n){ }^{16} \mathrm{O}$ during the interpulse period (Straniero et al. 1995), in contrast to the ${ }^{22} \mathrm{Ne}$ source, which operates during TPs. The timescales for neutron production during the interpulse are much longer $\left(\gtrsim 10^{3}\right.$ years $)$ than during the convective pulse ( $\sim 10$ years $)$, resulting in much lower neutron densities $\left(\sim 10^{7}\right.$ neutrons $\left.\mathrm{cm}^{-3}\right)$ than the ${ }^{22} \mathrm{Ne}$ source. Together, the timescales for neutron production and the neutron source determine the resulting $s$-process element distribution. The details of how the ${ }^{13} \mathrm{C}$ pocket forms and its extent in mass in the He-intershell are still unknown, although various mechanisms have been proposed, including convective overshoot, rotation, and gravity waves; see Herwig (2005) for a discussion of the relative merits of each mechanism.

The importance of the ${ }^{13} \mathrm{C}$ pocket and the ${ }^{13} \mathrm{C}(\alpha, n){ }^{16} \mathrm{O}$ reaction for nucleosynthesis in intermediate-mass stars is neither clear nor well studied. Adding a PMZ artificially into massive AGB models has little effect on the nucleosynthesis of nuclei lighter than $\mathrm{Fe}$ (Karakas et al. 2006) and on the production of $\mathrm{Ge}$ in $5 M_{\odot}, Z=0.02$ models (Karakas et al. 2007). The first point can be understood by noting that the ${ }^{13} \mathrm{C}$ pocket is about $10 \%$ of the mass of the He-intershell. Since the He-intershell is already smaller in massive AGB stars by about one order of magnitude compared to lower-mass stars, the inclusion of the PMZ has little effect on the surface abundances of light elements, which are relatively abundant in comparison to heavy elements produced via the $s$-process. The effect of the ${ }^{13} \mathrm{C}$ pocket on the production of $s$ nuclei is less clear, given their low initial abundances. Se and $\mathrm{Kr}$ elemental abundances derived from Type I PN spectra, in combination with observations of the elements $\mathrm{Rb}$ and $\mathrm{Zr}$ in massive AGB stars in the Galaxy, LMC, and SMC, may provide a way of distinguishing between the relative importance of the two neutron sources in intermediate-mass AGB stars.

For the $3,4,5$, and $6.5 M_{\odot}$ solar composition models, we present results with and without a PMZ (see Table 2 for the PMZ masses used in each model). No PMZ was included in any of the low-metallicity models, with the exception of the $2.5 M_{\odot}, Z=0.008$ model, where we only include results with a PMZ. The PMZ masses were chosen such that the resulting ${ }^{13} \mathrm{C}$ pockets were $\sim 10-15 \%$ of the mass of the He-intershell, consistent with the ${ }^{13} \mathrm{C}$ pocket masses used by Gallino et al. (1998). The method we use to include a PMZ is described in Lugaro et al. (2004) and is similar to that used by Goriely \& Mowlavi (2000). At the deepest extent of each TDU episode, we add an exponentially decaying proton profile that covers the mass specified in Table 2. This method is different from that used by Gallino et al. (1998), who included a ${ }^{13} \mathrm{C}$ profile directly into the intershell.

\section{MODEL RESULTS}

\subsection{Germanium to Strontium}

Surface abundances at the tip of the TP-AGB for $\mathrm{Zn}$ to $\mathrm{Sr}$ are presented in Table 2, and Figure 4 depicts enrichments of Se and $\mathrm{Kr}$ as a function of the pulse number in selected models. At solar metallicity, the $3 M_{\odot}$ models with a ${ }^{13} \mathrm{C}$ pocket produce the largest surface enrichments of $s$-process nuclei, with final $[\mathrm{Se} / \mathrm{Fe}]$ and $[\mathrm{Sr} / \mathrm{Fe}]$ abundances of $\sim 0.50$ and 0.85 for the $Z=0.02$ model, and 0.56 and 1.2 for the $Z=0.012$ model, respectively. In the lower metallicity models, the largest surface enrichments are seen in the $2.5 M_{\odot}, Z=0.008$ model with final $[\mathrm{Se} / \mathrm{Fe}]$ and $[\mathrm{Sr} / \mathrm{Fe}]$ abundances of 0.60 and 1.3, and in the $5 M_{\odot}, Z=0.004$ model with $\sim 0.90$ and 0.93, respectively.

The solar metallicity intermediate-mass $\left(M \geqslant 5 M_{\odot}\right)$ models exhibit little $s$-process enrichment $([\mathrm{Kr} / \mathrm{Fe}]$ and $[\mathrm{Sr} / \mathrm{Fe}]<$ 0.25). Using Asplund et al. (2005) solar abundances, the $6.5 \mathrm{M}_{\odot}$, $Z=0.012$ model shows slightly larger enhancements $([\mathrm{Kr} / \mathrm{Fe}]$ $=0.40,[\mathrm{Sr} / \mathrm{Fe}]=0.30)$, albeit only when a PMZ is added. The inclusion of a ${ }^{13} \mathrm{C}$ pocket has little effect $(\lesssim 0.1 \mathrm{dex})$ on the production of Se and Ge, as noted by Karakas et al. (2007), but it does enhance the $\mathrm{Kr}$ and $\mathrm{Sr}$ abundances. Only the lowermetallicity 5 and $6 M_{\odot}$ models produce substantial enrichments of $s$-process elements. These models experience a large number of TPs ( $\gtrsim 60)$, each with efficient TDU, resulting in significant surface enrichments. No ${ }^{13} \mathrm{C}$ pocket was used for these models, and thus it is clear that the higher temperatures obtained during TPs efficiently activate the ${ }^{22} \mathrm{Ne}$ neutron source. Moreover, the number of neutrons per seed nucleus is larger at lower metallicity owing to the smaller abundance of seed nuclei (e.g., ${ }^{56} \mathrm{Fe}$ ).

We find that $\mathrm{Kr}$ is enhanced in the stellar envelopes to a greater degree than $\mathrm{Se}$, in agreement with the observational results of Sterling et al. (2007) and SD08. This is because Kr lies nearer to the first $s$-process peak than $\mathrm{Se}$, and has an isotope $\left({ }^{86} \mathrm{Kr}\right)$ 
Table 2

Surface Abundance Results for Zn Through Sr, Taken at the Tip of the AGB from the Last Computed Model

\begin{tabular}{|c|c|c|c|c|c|c|c|c|c|}
\hline Mass & PMZ & {$[\mathrm{Zn} / \mathrm{Fe}]$} & {$[\mathrm{Ge} / \mathrm{Fe}]$} & {$[\mathrm{Se} / \mathrm{Fe}]$} & {$[\mathrm{Br} / \mathrm{Fe}]$} & {$[\mathrm{Kr} / \mathrm{Fe}]$} & $\delta^{86} \mathrm{Kr} /{ }^{82} \mathrm{Kr}^{\mathrm{a}}$ & {$[\mathrm{Sr} / \mathrm{Fe}]$} & $\Delta \mathrm{Fe}^{\mathrm{b}}$ \\
\hline \multicolumn{10}{|c|}{$Z=0.02$, Anders \& Grevesse (1989) C, N, and O } \\
\hline 3.0 & 0.0 & 0.001 & 0.004 & 0.004 & $<-0.001$ & 0.006 & -56.05 & 0.007 & $<0.001$ \\
\hline 3.0 & 0.001 & 0.071 & 0.263 & 0.346 & 0.299 & 0.477 & -693.3 & 0.700 & -0.00152 \\
\hline 3.0 & 0.002 & 0.122 & 0.397 & 0.493 & 0.433 & 0.628 & -774.4 & 0.853 & -0.0021 \\
\hline 4.0 & 0.0 & 0.004 & 0.011 & 0.009 & 0.003 & 0.010 & -25.82 & 0.007 & -0.0016 \\
\hline 4.0 & $1(-4)$ & 0.077 & 0.282 & 0.365 & 0.282 & 0.519 & -40.31 & 0.587 & -0.0026 \\
\hline 5.0 & 0.0 & 0.012 & 0.031 & 0.026 & 0.011 & 0.028 & -10.85 & 0.011 & -0.0024 \\
\hline 5.0 & $1(-4)$ & 0.024 & 0.096 & 0.132 & 0.083 & 0.241 & 307.7 & 0.246 & -0.0024 \\
\hline $5.0^{\mathrm{c}}$ & 0.0 & 0.073 & 0.188 & 0.181 & 0.107 & 0.210 & 91.56 & 0.085 & -0.0090 \\
\hline 6.0 & 0.0 & 0.038 & 0.106 & 0.104 & 0.051 & 0.131 & 92.09 & 0.055 & -0.0038 \\
\hline 6.5 & 0.0 & 0.047 & 0.133 & 0.138 & 0.064 & 0.187 & 186.3 & 0.083 & -0.0037 \\
\hline \multicolumn{10}{|c|}{$Z=0.012$, Asplund et al. (2005) $\mathrm{C}, \mathrm{N}$, and $\mathrm{O}$} \\
\hline 3.0 & 0.002 & 0.106 & 0.434 & 0.566 & 0.473 & 0.645 & -747.2 & 1.232 & -0.0028 \\
\hline 6.5 & 0.0 & 0.042 & 0.113 & 0.111 & 0.054 & 0.136 & 55.60 & 0.053 & -0.0047 \\
\hline 6.5 & $1(-4)$ & 0.052 & 0.164 & 0.203 & 0.122 & 0.397 & 1171 & 0.300 & -0.0048 \\
\hline \multicolumn{10}{|c|}{$Z=0.008$} \\
\hline 2.5 & 0.002 & 0.148 & 0.478 & 0.600 & 0.547 & 0.794 & -722.8 & 1.31 & -0.0040 \\
\hline 5.0 & 0.0 & 0.163 & 0.380 & 0.401 & 0.302 & 0.506 & 355.3 & 0.264 & -0.0138 \\
\hline 6.0 & 0.0 & 0.179 & 0.432 & 0.476 & 0.324 & 0.657 & 712.7 & 0.388 & -0.0098 \\
\hline \multicolumn{10}{|c|}{$Z=0.004$} \\
\hline 5.0 & 0.0 & 0.403 & 0.813 & 0.897 & 0.734 & 1.176 & 1178 & 0.927 & -0.0216 \\
\hline
\end{tabular}

Notes.

${ }^{\text {a }} \delta\left({ }^{i} X /{ }^{\text {ref }} X\right)$ notation is defined according to $\left[\left({ }^{i} X /{ }^{\text {ref }} X\right) /\left({ }^{i} X /{ }^{\text {ref }} X\right)_{\odot}-1\right] \times 10^{3}$, where ${ }^{\text {ref }} X$ is a reference isotope.

${ }^{\mathrm{b}}$ Defined by $\log \left(Y_{\text {final }} / Y_{\text {initial }}\right)$, where $Y$ is the surface abundance in number fraction.

${ }^{c}$ The model has Reimers mass loss on the AGB instead of Vassiliadis \& Wood (1993).

with a magic number of neutrons. ${ }^{10}$ Furthermore, the larger $s$-process enhancement of $\mathrm{Kr}$ relative to $\mathrm{Se}$ in the AGB models are in agreement with the chemical evolution results of Travaglio et al. (2004), who found that the main s-process contributions of AGB stars to the solar system $\mathrm{Se}$ and $\mathrm{Kr}$ abundances are $14 \%$ and $30 \%$, respectively (the weak $s$-process, operative in massive stars, contributes $25 \%$ and $20 \%$ to the solar Se and $\mathrm{Kr}$ abundances, respectively, while the $r$-process provides the remainder).

The $[\mathrm{Ge} / \mathrm{Fe}]$ abundances in Table 2 are different from those given in Table 2 of Karakas et al. (2007), owing to small changes to the neutron-capture cross sections used in the 166 and 156 species networks. There is a 0.08 dex change in the $[\mathrm{Ge} / \mathrm{Fe}]$ abundance for the $3 M_{\odot}, Z=0.02$ model with a PMZ of $0.002 M_{\odot}$. This difference of 0.08 dex is less than found when considering other model uncertainties. For example, there is a 0.16 dex difference in the $[\mathrm{Ge} / \mathrm{Fe}]$ abundances from the two different $5 M_{\odot}, Z=0.02$ models with different mass-loss laws.

Efficient activation of the ${ }^{22} \mathrm{Ne}$ neutron source leads to positive $\delta^{86} \mathrm{Kr} /{ }^{82} \mathrm{Kr}$ ratios, ${ }^{11}$ as can be seen in Table 2 for the most massive AGB models. These positive $\delta^{86} \mathrm{Kr} /{ }^{82} \mathrm{Kr}$ ratios are a result of neutron densities in excess of $10^{8} \mathrm{~cm}^{-3}$, which enables neutron captures on ${ }^{85} \mathrm{Kr}$ that produce ${ }^{86} \mathrm{Kr}$. If the density is lower, as is the case when ${ }^{13} \mathrm{C}(\alpha, n){ }^{16} \mathrm{O}$ is the neutron source, the branching is bypassed and ${ }^{85} \mathrm{Rb}$ is produced instead, resulting in negative values of the $\delta^{86} \mathrm{Kr} /{ }^{82} \mathrm{Kr}$ ratio. The $Z=0.02$ models reflect this trend, showing an increase in the $\delta^{86} \mathrm{Kr} /$

\footnotetext{
${ }^{10}$ Nuclei with a magic number of neutrons are relatively stable against $n$-capture due to their small $n$-capture cross-sections and are the cause of the peaks in the $s$-process enrichment distribution.

11 The $\delta$ notation is commonly used in meteoritics (see, e.g., Lugaro et al. 2003a), and is a variation from solar in parts permil, or parts per thousand (where a 100 permil change in the isotopic value is equivalent to a $10 \%$ variation).
}

${ }^{82} \mathrm{Kr}$ ratio with mass, with $5 M_{\odot}$ being the minimum mass for efficient activation of the ${ }^{22} \mathrm{Ne}$ source. The ratio is negative in the 3-4 $M_{\odot}$ models, where the ${ }^{13} \mathrm{C}$ reaction dominates as the neutron source and the ${ }^{22} \mathrm{Ne}$ reaction is only marginally activated. Values of $\delta\left({ }^{86} \mathrm{Kr} /{ }^{82} \mathrm{Kr}\right)$ measured in meteoritic stellar $\mathrm{SiC}$ grains from AGB stars roughly range from -500 to 900 and increase with the grain size (Lewis et al. 1994). Negative values are well explained by AGB model predictions; however, it is difficult to explain the large observed positive values because they are only produced by HBB models, where the $C>O$ condition necessary for the formation of $\mathrm{SiC}$ grains is not attained, except in the lowest metallicity, $Z=0.004$ models. Further detailed work is required on this issue.

The resulting elemental abundance pattern also differs, as $\mathrm{Sr}$ is produced in favor of $\mathrm{Rb}$ in the low-neutron density case, resulting in low $\mathrm{Rb} / \mathrm{Sr}$ ratios $(\sim 0.05)$. This is consistent with observations of low-mass AGB stars (Lambert et al. 1995; Abia et al. 2001). In the high-neutron density case, $\mathrm{Rb}$ is produced instead, as observed in massive O-rich AGB stars (GarcíaHernández et al. 2006). Preliminary results for $\mathrm{Zr}$ and $\mathrm{Rb}$ from intermediate-mass AGB stars are discussed in van Raai et al. (2008), and will be presented in more detail in a forthcoming paper (M.A. van Raai et al. 2008, in preparation).

\subsection{The Iron-Peak Elements Iron and Zinc}

In Table 2, we show the $[\mathrm{Zn} / \mathrm{Fe}]$ ratios and $\Delta \mathrm{Fe}$ values for each model, where $\Delta \mathrm{Fe}$ is the logarithmic change to the surface iron abundance from the main sequence to the tip of the TP-AGB. The $\Delta \mathrm{Fe}$ values are all very nearly zero, indicating that the surface abundance of this element is unaltered by AGB nucleosynthesis. The maximum effect among the $Z=0.02$ models is $2 \%$, and even for the $Z=0.004$ case, the Fe abundance 

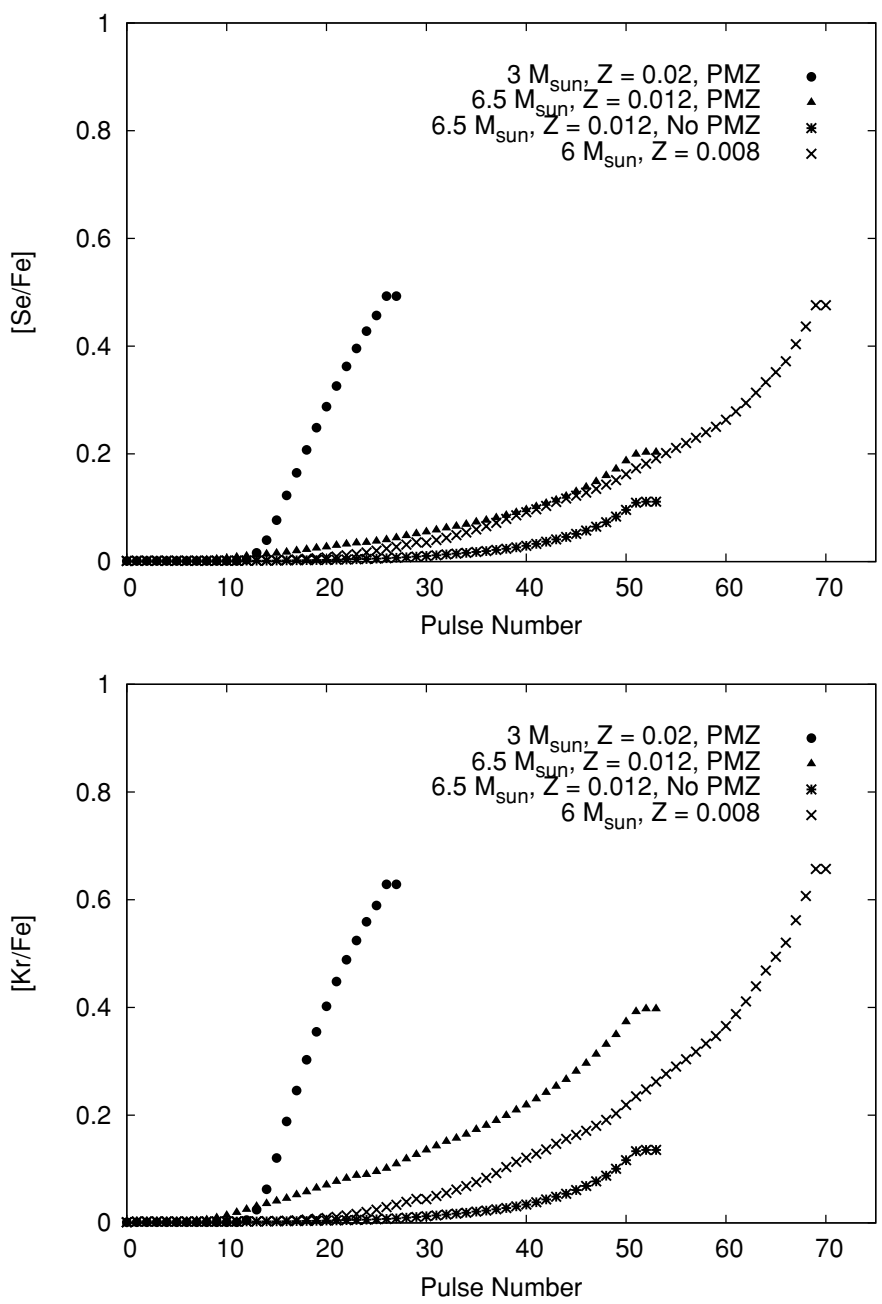

Figure 4. We show the $[\mathrm{Se} / \mathrm{Fe}]$ ratio (top panel) and the $[\mathrm{Kr} / \mathrm{Fe}]$ ratio (bottom panel), as a function of the thermal pulse number, from a selected number of stellar models. Each point represents the surface abundance during the interpulse period.

decreases by less than $5 \%$. This makes Fe a suitable reference element for estimating metallicities in AGB stars.

Although the surface $\mathrm{Fe}$ abundance is not significantly changed by nucleosynthesis during the AGB, the abundance of $\mathrm{Fe}$ in the He-intershell can be depleted by up to $\sim 1$ dex in a massive AGB star (e.g., $6.5 M_{\odot}$ model). In the intershell, ${ }^{56} \mathrm{Fe}$ is destroyed by neutron captures, and as the dominant isotope, it primarily determines the elemental abundance (even if the abundances of the rare neutron-rich isotopes ${ }^{57} \mathrm{Fe}$ and ${ }^{58} \mathrm{Fe}$ increase). In lower mass models (e.g., $3 M_{\odot}$ ), depletion of $\mathrm{Fe}$ is restricted to the ${ }^{13} \mathrm{C}$ pocket that encompasses $10-15 \%$ of the mass of the intershell. The next TP homogenizes this small, Fe-depleted region with the much larger Fe abundance in the rest of the shell. Our $3 M_{\odot}$ results are consistent with those of Werner \& Herwig (2006), who found that AGB models cannot account for the large Fe depletions found in some central stars of PNe, particularly the hot, H-deficient stars of the PG1159 class (Miksa et al. 2002; Rauch et al. 2008). Werner \& Herwig (2006) estimated that these central stars have mean masses of $\sim 0.62 M_{\odot}$, and thus represent the evolutionary remnants of lower-mass progenitors than those addressed here, and should be compared with models of stars with $\lesssim 3 M_{\odot}$. Note that similar or even more extreme $\mathrm{Fe}$ deficiencies-along with dramatic overabundances of $n$-capture elements-are seen in older white dwarfs where mechanisms such as gravitational settling and levitation by selective radiation pressure play important roles in establishing the surface abundances (Chayer et al. 2005; Werner \& Herwig 2006).

In PNe, Fe can be depleted into dust by large factors that may vary from object to object (Perinotto et al. 1999), and even in different regions within the same nebula (Sterling et al. 2005), so that its gas-phase abundance is not a reliable indicator of the true elemental abundance. The heavier iron group element $\mathrm{Zn}$ exhibits much milder depletions in the ISM, particularly in the warm phases (e.g., Welty et al. 1999). Consequently, Zn is frequently used as a proxy for iron and the iron group, for example in damped Lyman- $\alpha$ absorbers (e.g., Akerman et al. 2005). However, the use of $\mathrm{Zn}$ as an indicator of the initial composition of an AGB star or PN requires that the change in the abundance of $\mathrm{Zn}$ due to internal nucleosynthesis is small compared to its initial abundance in the star. It can be seen from Table 2 that the predicted changes in the $\mathrm{Zn}$ abundance are modest or negligible for most of the models. This is consistent with the conclusions of Travaglio et al. (2004), who estimated the total contribution of AGB stars to the solar $\mathrm{Zn}$ to be $\sim 3 \%$, and with Cayrel et al. (2004)'s finding that Zn production in the Milky Way is dominated by other sources (i.e., explosive Si burning in massive stars). However, at low metallicities, the models show a large enough increase that the $\mathrm{Zn}$ enrichment may be detectable.

These results can be compared to the $\mathrm{Zn}$ abundances derived for two Type I PNe (DGS08). Of the models presented here, the Galactic disk PN M 1-40 is most closely matched by the 6.0 $M_{\odot}$ model with $Z=0.02$, given its solar $\mathrm{Ne}$ and $\mathrm{S}$ abundances and $\mathrm{N} / \mathrm{O}=1.13$ (Górny et al. 2004). This model predicts a very small enrichment of $\mathrm{Zn},[\mathrm{Zn} / \mathrm{Fe}] \sim 0.038$, which is consistent with the measured value (see Section 2), even allowing for a possible small depletion effect. The case of LMC SMP 62 is more problematical. According to its $\alpha$-element abundances, the most appropriate models are those for $Z=0.008$. At this metallicity, all three of the models in Table 2, calculated for 2.5, 5.0, and 6.0 $M_{\odot}$ respectively, predict similar $\mathrm{Zn}$ enhancements of $\sim 0.15-0.2$ dex. However, as described in Section 2, the measured $\mathrm{Zn}$ abundance in this PN is lower, rather than higher, than those of the $\alpha$-elements (e.g., $\mathrm{Ne}, \mathrm{S}$ ), in contradiction to the models. The disagreement is even worse for Se, which is predicted to be enhanced by $0.4-0.6$ dex, depending on the initial mass. A modest enrichment in both $\mathrm{Zn}$ and $\mathrm{Se}$ is possible, if their initial abundances were low enough relative to $\mathrm{Fe}$, but, nevertheless, it remains difficult to satisfy the observational constraints for LMC SMP 62 with the listed models. We note, however, that these particular models also predict $\mathrm{N} / \mathrm{O}$ values either lower (the $2.5 M_{\odot}$ model) or higher (the 5 and $6 M_{\odot}$ models) than the observed value for this PN; in Section 5, we discuss other possible scenarios for explaining the observed properties of LMC SMP 62.

\subsection{Neon Through Argon}

Abundances of $\mathrm{S}, \mathrm{Cl}$, and $\mathrm{Ar}$ in $\mathrm{PNe}$ are powerful tools for studying the chemical evolution of galaxies and stellar systems, under the assumptions that these elements are unaltered by AGB evolution (Dopita et al. 1997; Stasińska et al. 1998; Leisy \& Dennefeld 2006; Idiart et al. 2007) and are produced in the same relative proportions in massive stars (Stasińska et al. 1998). The case of $\mathrm{Ne}$ is not as clear, although the remarkably constant $\mathrm{Ne} / \mathrm{O}$ ratio of $\sim 0.20$ (by mass) observed in $\mathrm{PNe}$ with a wide 
Table 3

Abundance Results for $\mathrm{O}, \mathrm{Ne}, \mathrm{P}, \mathrm{S}, \mathrm{Cl}$, and $\mathrm{Ar}$

\begin{tabular}{lccccccccc}
\hline \hline Mass & $Z$ & $\epsilon(\mathrm{O})^{\mathrm{a}}$ & $\epsilon(\mathrm{Ne})$ & $\mathrm{Ne} / \mathrm{O}^{\mathrm{b}}$ & $\epsilon(\mathrm{P})$ & $\epsilon(\mathrm{S})$ & $\epsilon(\mathrm{Cl})$ & $\epsilon(\mathrm{Ar})$ & $\delta^{38} \mathrm{Ar}^{36} \mathrm{Ar}^{\mathrm{c}}$ \\
\hline Initial & 0.02 & 8.935 & 8.103 & 0.181 & 5.591 & 7.277 & 5.143 & 6.570 & 0.000 \\
3.0 & No PMZ & 8.940 & 8.279 & 0.273 & 5.595 & 7.299 & 5.192 & 6.592 & 7.735 \\
4.0 & No PMZ & 8.933 & 8.204 & 0.234 & 5.613 & 7.294 & 5.190 & 6.586 & 14.55 \\
5.0 & No PMZ & 8.931 & 8.191 & 0.227 & 5.617 & 7.310 & 5.208 & 6.603 & 17.76 \\
6.0 & No PMZ & 8.908 & 8.168 & 0.227 & 5.696 & 7.328 & 5.237 & 6.621 & 26.29 \\
6.5 & No PMZ & 8.905 & 8.166 & 0.228 & 5.635 & 7.330 & 5.212 & 6.623 & 25.18 \\
Initial & 0.012 & 8.659 & 7.838 & 0.189 & 5.358 & 7.139 & 5.498 & 6.178 & 0.000 \\
3.0 & No PMZ & 8.656 & 8.086 & 0.336 & 5.393 & 7.165 & 5.530 & 6.205 & 17.00 \\
& 0.002 & 8.666 & 8.666 & 0.486 & 5.522 & 7.165 & 5.534 & 6.204 & 41.34 \\
6.5 & No PMZ & 8.563 & 7.868 & 0.277 & 5.410 & 7.188 & 5.548 & 6.227 & 61.39 \\
Initial & 0.008 & 8.514 & 7.675 & 0.181 & 5.155 & 6.849 & 4.715 & 6.142 & 0.000 \\
2.5 & No PMZ & 8.521 & 7.846 & 0.431 & 5.178 & 6.870 & 4.754 & 6.162 & 16.37 \\
& 0.002 & 8.528 & 7.985 & 0.653 & 5.251 & 6.867 & 4.762 & 6.160 & 32.81 \\
5.0 & No PMZ & 8.419 & 7.817 & 0.313 & 5.454 & 6.899 & 4.886 & 6.192 & 92.67 \\
6.0 & No PMZ & 8.317 & 7.781 & 0.364 & 5.471 & 6.908 & 4.861 & 6.201 & 63.44 \\
Initial & 0.004 & 8.206 & 7.367 & 0.181 & 4.847 & 6.542 & 4.407 & 5.835 & 0.000 \\
5.0 & No PMZ & 8.052 & 7.665 & 0.512 & 5.444 & 6.609 & 4.633 & 5.900 & 136.7 \\
\hline
\end{tabular}

Notes.

a $\epsilon(\mathrm{Y})=\log 10(Y / H)+12$, where abundances are measured by number, and $H$ is the abundance of hydrogen.

$\mathrm{b}$ The $\mathrm{Ne} / \mathrm{O}$ ratio is calculated by mass, to allow comparison to the observations.

c The $\delta$ notation was previously defined in Table 2 .

variety of chemical compositions indicates that, in most cases, this ratio is not altered during the AGB. ${ }^{20} \mathrm{Ne}$, the most abundant isotope of $\mathrm{Ne}$, is not altered significantly by $\mathrm{H}$ or $\mathrm{He}-$ burning in AGB stars, whereas ${ }^{22} \mathrm{Ne}$ can be produced by successive $\alpha$-captures onto ${ }^{14} \mathrm{~N}$. Therefore, an enhancement in the $\mathrm{Ne} / \mathrm{O}$ ratio is expected only when ${ }^{22} \mathrm{Ne}$ is sufficiently enriched that its abundance is comparable to or exceeds that of ${ }^{20} \mathrm{Ne}$. Karakas \& Lattanzio (2003b) predicted that such an increase would only occur in a narrow range of progenitor masses, $\sim 2.5$ to $\sim 3.5 M_{\odot}$ depending on metallicity. Our current models are generally consistent with these earlier results, although from Table 3, it is clear that the $6.5 M_{\odot}, Z=0.012$ model and the 5 and $6 M_{\odot}, Z=0.008$ and 0.004 models have enhanced $\mathrm{Ne} / \mathrm{O}$ ratios as a result of some $\mathrm{O}$ destruction by $\mathrm{HBB}$ and mild enrichments of $\mathrm{Ne}$ via dredge-up (maximum $\mathrm{Ne}$ increase of $\sim 0.3$ dex for the 5, $6 M_{\odot}$ metal-poor models). Therefore, Type I PNe are not expected to be significantly enriched in Ne except at low metallicities.

${ }^{22} \mathrm{Ne}$ is produced in the TPs themselves, but in lower mass models $\left(2.5\right.$ and $3 M_{\odot}$ ), the partial mixing of protons can further enhance the $\mathrm{Ne} / \mathrm{O}$ ratio. This is because the partially mixed zone results in a pocket of ${ }^{13} \mathrm{C}$ and ${ }^{14} \mathrm{~N}$, thus increasing the ${ }^{14} \mathrm{~N}$ abundance in the intershell to values higher than that from $\mathrm{CN}$-cycling during the interpulse. The next TP quickly converts ${ }^{14} \mathrm{~N}$ into ${ }^{22} \mathrm{Ne}$. The resulting ${ }^{22} \mathrm{Ne}$ abundances after the TP are slightly higher than in the case with no partially mixed zone. After many TPs and TDU, the PMZ results in an increase in the elemental $\mathrm{Ne}$ abundance. The observations by Bernard-Salas et al. (2008) are intriguing in this respect. They determined Ne/S ratios in a sample of $25 \mathrm{PNe}$ in the Magellanic Clouds, and found that four of the objects show high $\mathrm{Ne} / \mathrm{S}$ ratios. For two of the objects, they attribute the high ratios to low $\mathrm{S}$ abundances, but in the other two PNe, the high $\mathrm{Ne} / \mathrm{S}$ values could be due to $\mathrm{Ne}$ enrichments.

Table 3 shows the surface abundance predictions for $\mathrm{S}, \mathrm{Cl}$, and Ar. These elements are generally not altered by AGB nucleosynthesis processes by more than the observational errors $(\sim \pm 0.1-0.15 \mathrm{dex})$, consistent with the observations of $\mathrm{PNe}$ by Stasińska et al. (1998). Neutron captures in the He-shell can result in variations in the isotopic ratios involving the stable $\mathrm{Cl}$ and $\mathrm{Ar}$ isotopes; this is demonstrated in the last column of Table 3, where we show the permil variations for ${ }^{38} \mathrm{Ar} /{ }^{36} \mathrm{Ar}$. Values of $\delta\left({ }^{38} \mathrm{Ar} /{ }^{36} \mathrm{Ar}\right)$ measured in stellar $\mathrm{SiC}$ grains range from zero (solar composition) to positive values up to $\sim 200$ (Lewis et al. 1994), in qualitative agreement with our model predictions. Kahane et al. (2000) compared AGB model predictions to measurements of $\mathrm{Cl}, \mathrm{Mg}, \mathrm{Si}$, and $\mathrm{S}$ isotopic ratios in the cool carbon star IRC +10216 , and inferred an initial mass of $\lesssim 2 M_{\odot}$ based on the accuracy of the $\mathrm{Cl}$ and $\mathrm{Mg}$ data. Kahane et al. concluded that a larger mass progenitor would lead to too high $\mathrm{Cl}$ and $\mathrm{Mg}$ isotopic ratios at the surface, although our models suggest that progenitor masses as high as $\sim 3 M_{\odot}$ produce a good fit to the observed data, within the errors quoted for the isotopic ratios. In the case of our low-metallicity massive AGB models, neutron captures result in modest increases $(\sim 0.25 \mathrm{dex})$ in the elemental $\mathrm{Cl}$ surface abundance.

The phosphorous abundance can be determined in hot postAGB objects such as the PG 1159 stars, whose envelopes are composed of He-intershell material. Observations indicate that PG 1159 stars have solar P abundances, whereas $3 M_{\odot}$ models predict $\mathrm{P}$ abundances ranging from four times solar to $\sim 25$ times solar in the He-intershell, depending on the amount of extra mixing included in the calculations to produce the ${ }^{13} \mathrm{C}$ pocket (Werner \& Herwig 2006). Our He-shell predictions are consistent with Herwig's model, in that we estimate $\mathrm{P}$ enhancements from 1.3 to $\sim 9$ times solar for the $3 M_{\odot}$ model, without and with a PMZ, respectively. The large P abundances in the He-intershell abundances do not result in large surface enrichments ( $\lesssim 0.1 \mathrm{dex}$ in all solar metallicity models), so that if measurements could be made of the P abundances in PNe, we would not expect to see variations from solar.

Sulfur is also observed in PG 1159 stars, with abundances varying from 0.01 times solar to solar (Werner \& Herwig 2006). It is not clear if this range reflects the initial abundance of the star, various degrees of nuclear processing, condensation of $S$ into dust, or problems in determining accurate $S$ abundances. 
Note that $\mathrm{S}$ abundances in several Galactic (Henry et al. 2004) and some Magellanic Cloud PNe (Bernard-Salas et al. 2008) are low relative to $\mathrm{O}$. While IR observations of $\mathrm{S}^{3+}$ in Galactic PNe seem to indicate that uncertainties in corrections for unobserved ionization stages are not the reason for the low $\mathrm{S}$ abundances, it is possible that $\mathrm{S}$ may be depleted into dust in some PNe (especially C-rich objects; Pottasch \& Bernard-Salas 2006). AGB models do not predict large depletions of $\mathrm{S}$ in the Heshell, with $\mathrm{S}$ abundances varying between 0.6 and 0.9 times solar (Werner \& Herwig 2006). Our values lie from no change to $\sim 0.9$ times solar. The results for $\mathrm{P}$ and $\mathrm{S}$ are puzzling, indicating, on the one hand, that a smaller PMZ is required (to produce less $\mathrm{P}$ ) or, on the other hand, that more mixing is required (to destroy more S).

\subsection{Oxygen in Intermediate-Mass AGB Stars}

Oxygen constitutes a large fraction of the total metal content of a star, and in this sense is a more fundamental measure of metallicity than $\mathrm{Fe} / \mathrm{H}$, which has been historically used as the primary indicator of metallicity due to its ease of measurement. While it can be challenging to determine $\mathrm{O}$ abundances in stars (e.g., Ramírez et al. 2007), O/H is easily measured in ionized nebulae because of its bright emission lines, and the resulting abundances are considered to be among the most accurate for any element. According to the current paradigm for galactic chemical evolution, $\mathrm{O}$ production is dominated by massive stars $\left(M \geqslant 10 M_{\odot}\right)$. Nucleosynthesis models of AGB stars indicate that, collectively, these stars do not significantly contribute to the production of O (Forestini \& Charbonnel 1997; Karakas \& Lattanzio 2007), although there is evidence for dredge-up of self-produced oxygen in a few special cases, including PNe from metal-poor progenitor stars (e.g., Péquignot et al. 2000; Dinerstein et al. 2003). The inclusion of diffusive convective overshoot at the inner edge of the flash-driven convective zone during a TP can lead to $\mathrm{O}$ abundances in the intershell of $\sim 20 \%$ (Herwig 2000), in contrast to standard models that predict $2 \%$ by mass or less (Boothroyd \& Sackmann 1988; Karakas et al. 2002). This can lead to increases in the surface composition of $\mathrm{O}$, particularly at low metallicities. Evidence for this overshoot comes from PG 1159 stars that show compositions consistent with Herwig's models (Werner \& Herwig 2006). It is unclear, however, if this overshooting occurs for all AGB stars or only for AGB stars that produce PG 1159 stars as a consequence of late and very late TPs. For example, the observed abundance analyses in intrinsic and extrinsic AGB stars, as well as in $\mathrm{SiC}$ grains, suggest that such an overshoot is uncommon (Lugaro et al. 2003b). This is because in the region of the He-rich intershell where primary $\mathrm{O}$ is synthesized, the temperature is large enough for efficient activation of the ${ }^{22} \mathrm{Ne}(\alpha, n)^{25} \mathrm{Mg}$ neutron source, even in low-mass AGB stars. In this case, the resulting neutron density is very large (up to $\sim 10^{12}$ neutrons $\mathrm{cm}^{-3}$ ) in contrast to observations of low-mass AGB stars that indicate neutron densities of about $\sim 10^{7}$ neutrons $\mathrm{cm}^{-3}$ (Abia et al. 2001).

Intermediate-mass $\mathrm{AGB}$ stars destroy $\mathrm{O}$ via $\mathrm{HBB}$ when the temperature at the base of the convective envelope is sufficiently high $\left(T \gtrsim 80 \times 10^{6} \mathrm{~K}\right)$. The $\mathrm{O}-\mathrm{Na}$ anticorrelation, observed in globular clusters stars, has in fact been associated with HBB in intermediate-mass AGB stars, because HBB can qualitatively account for the destruction of $\mathrm{O}$ along with the production of $\mathrm{Na}$. Moreover, SD08 noted that the $\mathrm{Ar} / \mathrm{O}, \mathrm{S} / \mathrm{O}$, and $\mathrm{Cl} / \mathrm{O}$ ratios of Type I PNe are approximately a factor of 2 larger on average than in non-Type $\mathrm{I}$ objects. Since $\mathrm{Ar}, \mathrm{S}$, and $\mathrm{Cl}$ are not significantly affected by nucleosynthesis in low- or intermediate-mass stars (see Section 4.3), they interpreted this as evidence for $\mathrm{O}$ destruction during HBB in Type I PN progenitors. This result had also been found in previous investigations (Marigo et al. 2003; Leisy \& Dennefeld 2006; Pottasch \& Bernard-Salas 2006).

The amount of $\mathrm{O}$ destroyed by HBB depends on the temperature at the base of the convective envelope. The temperature, in turn, depends on a number of factors including the initial mass and metallicity, with hotter temperatures found in models of increasing mass for a given $Z$ or in models of decreasing metallicity at a given mass. In addition, the predicted efficiency of HBB is sensitive to the treatment of convection and mass loss in the models, neither of which are empirically well constrained. In particular, the convective efficiency affects the luminosity and the mass-loss rate (Ventura \& D'Antona 2005), and hence these properties are sensitive to the convective model that is utilized. Mass loss determines the length of the HBB phase, since HBB does not operate once the envelope mass drops below about $1.5 M_{\odot}$.

In Table 3, we present the $\mathrm{O}$ abundances at the surface of the model star at the tip of the AGB. The $\mathrm{O}$ abundance remains unaltered in the lower mass models ( 3 and $4 M_{\odot}, Z=0.02$ ), whereas $\mathrm{O}$ destruction takes place in models with HBB. Out of the $Z=0.02$ models, the largest amount of $\mathrm{O}$ destruction is seen at $6.5 M_{\odot}$, which has a peak temperature at the base of the envelope of $86 \times 10^{6} \mathrm{~K}$. The effect of metallicity is seen most clearly by comparing the $6.5 M_{\odot}$ models with $Z=0.02$ and $Z=0.012$, with the latter model computed assuming the Asplund et al. (2005) C, N, O initial abundances. In the $Z=0.012$ model the peak temperature is higher, resulting in 0.15 dex destruction of $\mathrm{O}$ compared to only 0.09 dex in the $Z=0.02$ model. Note that the $\mathrm{O}$ that is destroyed is all converted into $\mathrm{N}$. The largest $\mathrm{O}$ destruction of 0.26 dex occurs in the $6 M_{\odot}, Z=0.008$ model. Overall, none of the models suffered $\mathrm{O}$ destruction of more than 0.3 dex.

It is not clear that the low $\mathrm{O}$ abundances relative to $\mathrm{S}, \mathrm{Ar}$, and $\mathrm{Cl}$ in Type I PNe can be attributed to HBB. While modeling uncertainties in the treatment of convection and mass loss could, in principle, allow for more efficient $\mathrm{O}$ destruction during $\mathrm{HBB}$ than we report, this inevitably leads to large increases in the N/O ratio. This result agrees with the findings of Marigo et al. (2003), whose synthetic models with efficient ON-cycling produced $\mathrm{N} / \mathrm{O}$ ratios considerably higher than those observed in Type I PNe. These authors suggested that the low $\mathrm{O}$ abundances may be explained if the initial metallicities of Type I PN progenitors are low. However, this does not explain the approximately solar abundances of $\mathrm{S}$ and $\mathrm{Ar}$ in these objects. Moreover, our models constrain the metallicities of Type I PN progenitors to be $Z \gtrsim 0.4 Z_{\odot}$ in order to reproduce the observed $\mathrm{Se}$ and $\mathrm{Kr}$ abundances. This important issue remains unresolved.

\section{DISCUSSION}

In Figure 5, we illustrate the $s$-process abundance predictions from a selection of the stellar models. The gray-hatched box represents the approximate region occupied by Type I PNe $s$-process abundances. Typical observational uncertainties allow the maximum enrichment to reach $\approx 0.6 \mathrm{dex}$ in some cases. The intermediate-mass AGB models are, in general, a good match to the observed abundances of Se and $\mathrm{Kr}$ in Type I PNe. Models in Table 2, which show enhancements of Se and $\mathrm{Kr}$ greater than $0.5 \mathrm{dex}$, are the $2.5 M_{\odot}$ model, the $3 M_{\odot}$ models, the $6 M_{\odot}$, 


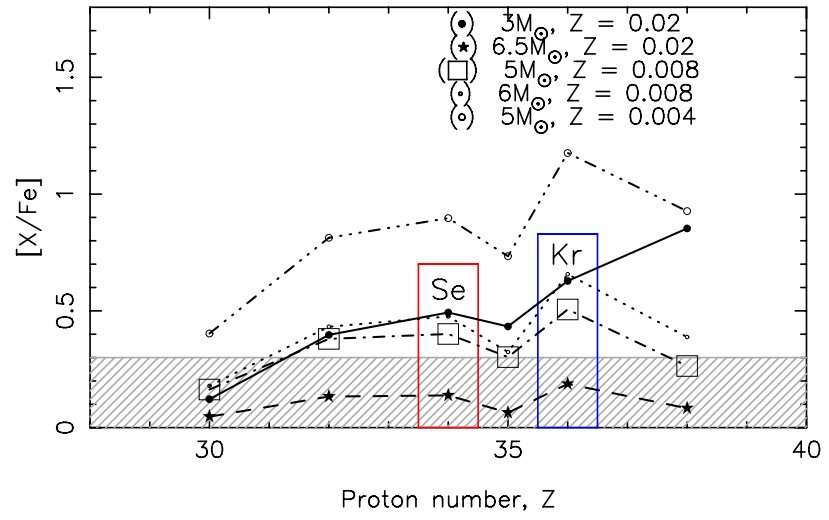

Figure 5. Surface abundances of $\mathrm{Zn}, \mathrm{Ge}, \mathrm{Se}, \mathrm{Br}, \mathrm{Kr}$, and $\mathrm{Sr}$ at the tip of the AGB phase for a selection of the stellar models. Abundances are shown as $[\mathrm{X} / \mathrm{Fe}]$ ratios, and are plotted as a function of the proton number, $Z$. The $3 M_{\odot}$, $Z=0.02$ model includes a PMZ of $0.002 M_{\odot}$ whereas all other models are without ${ }^{13} \mathrm{C}$ pockets. The gray-hatched box with a maximum at $[\mathrm{X} / \mathrm{Fe}]=$ 0.3 represents the region of observed Type I PN Se and $\mathrm{Kr}$ abundances. Note that observational uncertainties allow for $[\mathrm{Se}, \mathrm{Kr} / \mathrm{Fe}$ ] up to 0.6 . Boxes around the elements $\mathrm{Se}$ and $\mathrm{Kr}$ indicate that for the present study, we can only use these elements for comparison as large PN abundance datasets of the other trans-iron elements are not yet available.

(A color version of this figure is available in the online journal.)

$Z=0.008$ model, and the low-metallicity $5 M_{\odot}, Z=0.004$ model, which gives large enhancements for all elements between $\mathrm{Zn}$ and $\mathrm{Sr}$. The results for $3 M_{\odot}$ with a PMZ are only in borderline agreement with the observations, although the results with no PMZ are consistent with no $s$-process enrichments. In either case, the final composition is carbon-rich, indicating that this model is unlikely to be the precursor of a Type I PN. Indeed, the results for the $3 M_{\odot}$ models are closer to that found for the $2.5 M_{\odot}, Z=0.008$ model which has a distinct, non-Type I $\mathrm{PNe}$ composition, with a high final $\mathrm{C} / \mathrm{O}$ ratio, and considerable overabundances of all $s$-process elements $(>0.5$ dex for Se to Sr). The results for the $5 M_{\odot}, Z=0.004$ model suggest that Type I PNe in the Galaxy do not descend from low-metallicity stars, as suggested, for example, by Marigo et al. (2003), and that a minimum metallicity of $\sim 0.4 Z_{\odot}$ is required for these PNe. This result is not unexpected, given that Type I PNe evolve from more massive stars than other PNe, and hence trace a younger population. However, these model results suggest that Type I PNe in the Magellanic Clouds could be more highly enriched in light $n$-capture elements such as Se and $\mathrm{Kr}$ than their Galactic counterparts. This is a strong motivation for further observations of $s$-process elements in PNe belonging to the Magellanic Clouds or to other metal-poor populations.

Observations of other trans-iron elements in $\mathrm{PNe}$ in addition to Se and $\mathrm{Kr}$ could be used to constrain the mass and metallicity ranges of Type I PNe. For example, $\mathrm{Sr}$ and $\mathrm{Rb}$ abundances are potentially useful discriminants of progenitor mass. $\mathrm{Sr}$ is considerably enriched in the $3 M_{\odot}$ models ( $\left.[\mathrm{Sr} / \mathrm{Fe}] \sim 0.85-1.23\right)$, but is only slightly if at all enhanced in the 6 and $6.5 M_{\odot}$ models $([\mathrm{Sr} / \mathrm{Fe}]<0.4 \mathrm{dex})$. Note that the inclusion of a PMZ in the $6.5 M_{\odot}, Z=0.012$ model increases the level of Sr enrichment expected, from 0.05 to 0.3 dex.

Of these next two elements heavier than $\mathrm{Kr}$ (element number 36), $\mathrm{Rb}$ (element 37) is more likely to be measurable in a meaningful way in nebulae than $\mathrm{Sr}$ (element 38), for the following reasons. First, $\mathrm{Sr}$ is refractory and can be strongly depleted into dust grains. In fact, based on its condensation temperature (Lodders 2003), $\mathrm{Sr}$ is expected to be incorporated into dust to a greater degree than $\mathrm{Fe}$, whose gaseous abundance is depleted by 1-2 orders of magnitude in PNe (Perinotto et al. 1999; Sterling et al. 2005) and the ISM (Welty et al. 1999). Therefore, measurements of the gaseous Sr abundance in $\mathrm{PNe}$ are not likely to reveal useful information about $s$-process enrichments. Second, the only emission lines of $\mathrm{Sr}$ in the optical spectral region originate from relatively highly ionized states that are not expected to be abundant, except in a few of the highest-excitation PNe. Sr emission has been identified in only one PN to date (Pequignot \& Baluteau 1994; Zhang \& Liu 2005). On the other hand, based on its condensation temperature (Lodders 2003), Rb does not deplete into dust as readily as $\mathrm{Sr}$, and thus its gaseous abundance should be more representative of the overall elemental abundance. $\mathrm{Rb}$ emission has been identified in a number of PNe (Pequignot \& Baluteau 1994; Zhang \& Liu 2005; Sharpee et al. 2007) and should be detectable in $\mathrm{PNe}$ with a broader range of ionization than $\mathrm{Sr}$. This discussion suggests that $\mathrm{Rb}$ is a useful element with which to constrain stellar models to PN observations.

Furthermore, $\mathrm{Rb}$ is observed to be substantially enhanced in massive Galactic $\mathrm{OH} / \mathrm{IR}$ stars with the maximum enhancement $[\mathrm{Rb} / \mathrm{Fe}] \sim 2 \pm 1$ dex (García-Hernández et al. 2006). van Raai et al. (2008) have made a preliminary comparison between $\mathrm{Zr}$, $\mathrm{Rb}$, and $\mathrm{Li}$ predictions from 5 to $6.5 M_{\odot}$ solar composition models to the observations of the $\mathrm{OH} / \mathrm{IR}$ stars. The best match between stellar models and the observations was obtained with the $6.5 M_{\odot}$ model computed by (1) not including a ${ }^{13} \mathrm{C}$ pocket and (2) by accounting for extra TPs not modeled in detail, as done by Karakas et al. (2007). The extra TPs and TDU mixing episodes may occur because not all the envelope mass was lost when convergence difficulties ended the computation. The main uncertainty is the behavior of the TDU efficiency at small envelope masses, with studies generally finding a decrease in the efficiency with decreasing envelope mass (Straniero et al. 1997; Karakas et al. 2002). Further studies on the evolution of the TDU efficiency with decreasing envelope mass are needed to help settle this issue. The tabulated results presented in this study were calculated without the inclusion of these extra TPs and can be considered as lower limits to the final PN abundances, noting that it is highly uncertain how much of an impact the final TPs would have on the final PN abundances.

The final few TPs can potentially have a significant effect on the final abundance of the star and its resulting PN. For example, Frost et al. (1998) noted that intermediate-mass AGB stars may become luminous, optically obscured carbon stars near the end of the TP-AGB, when mass loss has removed much of the envelope, extinguishing HBB but allowing dredgeup to continue. Under these conditions, the envelope mass is significantly reduced, resulting in only minimal dilution of the intershell material that is dredged up. This can allow for large enhancements to the surface composition of $\mathrm{C}$ and other Heburning products. In the models with $\mathrm{HBB}$ included in this study, all experienced a few TPs and TDU episodes after the cessation of $\mathrm{HBB}$, with some of the lower metallicity models becoming carbon rich (see Table 1, i.e., $6 M_{\odot}, Z=0.008$; 5 and $6 M_{\odot}, Z=0.004$ ) before convergence difficulties ended the computation. Note that it is after this stage that we consider the effect of remaining TPs not modeled in detail using a synthetic AGB algorithm.

In van Raai et al. (2008), the inclusion of these remaining TPs only had a large impact on the surface abundances of the $6.5 M_{\odot}, Z=0.02$ model (out of the 5, 6, and 6.5 $M_{\odot}, Z=0.02$ models), and only when assuming that efficient TDU occurs at a 
very small envelope mass. ${ }^{12} \mathrm{~A}$ final $[\mathrm{Rb} / \mathrm{Fe}] \approx 0.9$ was obtained in the $6.5 M_{\odot}, Z=0.02$ model, in agreement with the observed $\mathrm{Rb}$ abundances of $\mathrm{OH} / \mathrm{IR}$ stars given the large uncertainties. Along with increases in $\mathrm{Rb}$, the [Se, $\mathrm{Kr} / \mathrm{Fe}$ ] abundances were increased to 0.6 and 0.7 dex (respectively), above the observed Type I PN limit of 0.3 dex. This model was estimated to have seven remaining TPs, with the last TP occurring with a very small envelope mass of $\sim 0.03 M_{\odot}$. The small envelope mass during the last TDU leads to the situation discussed above: a substantial increase in the surface abundances, caused by the mass of the envelope being only $\sim 10$ times greater than the mass of the intershell. It is questionable whether such an efficient TDU would occur for such a low envelope mass; so if we exclude this last mixing episode, the $[\mathrm{Se}, \mathrm{Kr} / \mathrm{Fe}]$ abundances are $\approx 0.4$ and 0.45 , respectively, in agreement with observed Type I PN abundances. This analysis may indicate that the OH/IR stars are not the progenitors of most Type I PNe and that there are possible observational biases in the sampling of each population. That is, the $\mathrm{OH} / \mathrm{IR}$ population may sample the most massive AGB stars $\left(6-7 M_{\odot}\right)$, with the largest $[\mathrm{Rb} / \mathrm{Fe}]$ (and $[\mathrm{Kr} / \mathrm{Fe}]$ ) abundances, while the Type I PN population may come from slightly less massive AGB stars, with lower $\mathrm{Kr}$ and $\mathrm{Rb}$ abundances. There is also the problem of explaining the large $\mathrm{Rb}$ abundances in the $\mathrm{OH} / \mathrm{IR}$ stars, given that they seem to require the TDU to efficiently operate at small envelope masses. More observations of $\mathrm{Rb}$ in $\mathrm{OH} / \mathrm{IR}$ stars and $\mathrm{PNe}$ will help to settle these issues.

Modeling uncertainties can dramatically affect AGB nucleosynthesis predictions. As previously noted, different convective models have a large impact on the nucleosynthesis of light elements (e.g., N, O, Na) in AGB models with HBB. Improvements in our understanding of convection are desperately needed, but this may only come about through multidimensional studies (e.g., Dearborn et al. 2006; Herwig et al. 2006; Meakin \& Arnett 2007). The mass-loss law used in the computations is similarly important, because this can determine (for a fixed convective model) the number of TPs and mixing episodes. For example, if we artificially cut the number of TPs by half for the $5 M_{\odot}$, $Z=0.004$ model (40 TPs instead of the computed 80), the final $[\mathrm{Kr} / \mathrm{Fe}]$ ratio is $\sim 0.35$ dex instead of 1.17 dex. This simple analysis indicates that we need better constraints on the mass loss from low-metallicity, massive AGB stars if we are to provide constraints on the metallicity range of Type I PNe.

Another important uncertainty relates to the unknown formation mechanism for ${ }^{13} \mathrm{C}$ pockets in AGB stars (Gallino et al. 1998; Herwig 2005). These pockets are necessary to provide neutrons in low-mass stars. It is unclear, however, what role the ${ }^{13} \mathrm{C}$ neutron source plays in more massive AGB stars. We had hoped to ascertain the dominant neutron source from comparisons of Type I PN abundances to massive AGB stellar models, but the Se and $\mathrm{Kr}$ abundances alone are not sufficient. Selenium abundances were not strongly affected by the inclusion of a ${ }^{13} \mathrm{C}$ pocket in the 5 and $6.5 M_{\odot}$ models, whereas the abundance of $\mathrm{Kr}$ was affected but not increased above 0.5 dex. Note that in three Type I PNe of SD08 where both Se and Kr were detected, an abundance of $[\mathrm{Kr} / \mathrm{Se}]=0.5$ was found, providing some support for the inclusion of a ${ }^{13} \mathrm{C}$ pocket in massive AGB models; this conclusion is, however, limited by small number statistics. We predict that PNe should have larger enrichments of $\mathrm{Kr}$ than Se. While this result is valid regardless of whether or not a ${ }^{13} \mathrm{C}$ pocket is included, larger $[\mathrm{Kr} / \mathrm{Se}]$ values are predicted in

\footnotetext{
12 Besides the inclusion of the extra TP, the models presented here and in
} van Raai et al. (2008) are the same. models with a PMZ (see Table 2). Firmer conclusions regarding the neutron source operating in intermediate-mass AGB stars may come from large data sets of many $n$-capture elements, including $\mathrm{Br}, \mathrm{Rb}$, and $\mathrm{Xe}$.

Finally, models with extra mixing may be necessary to reproduce the observed abundances of some PNe. For example, the composition determined for LMC SMP 62 (see Sections 2 and 4.2) suggests that it is a Type I PN, but its low Se and $\mathrm{Zn}$ abundances are at odds with our predicted abundances for intermediate-mass models. A $3 M_{\odot}, Z=0.02$ model with rotation by $M$. Cantiello \& N. Langer (2008, private communication) had an $\mathrm{N} / \mathrm{O}$ ratio of 0.43 after the first dredgeup, compared to 0.32 from our model with no rotation, whereas the N/O value of LMC SMP 62 is $\sim 0.4-0.5$. During core $\mathrm{H}$-burning, rotation mixes processed material to layers further out than they would otherwise be found. Rapid rotation may also inhibit the production of $s$-process elements during the AGB phase (Herwig et al. 2003), consistent with the lack of $\mathrm{Zn}$ and Se enrichments found in this object. An alternative is that LMC SMP 62 evolved from a low-mass progenitor of less than $2 M_{\odot}$, which did not experience efficient TDU. Note that the low C/O ratio of 0.17 found for this object (Aller et al. 1987) is also consistent with a star that did not experience any TDU mixing (suggesting an initial mass of $\lesssim 1.25 M_{\odot}$ ). It is unclear if rotation can explain the high $\mathrm{N} / \mathrm{O}$ ratio in such a low-mass star. Other extra-mixing processes (e.g., thermohaline mixing) may need to be invoked in this case.

\section{CONCLUSIONS}

Type I PNe have high $\mathrm{He} / \mathrm{H}$ and $\mathrm{N} / \mathrm{O}$ ratios, indicating that they are the descendants of intermediate-mass AGB stars with initial masses between $\sim 3$ and $8 M_{\odot}$. Sterling \& Dinerstein (2008) found that Type I PNe exhibited significantly smaller enrichments of Se and $\mathrm{Kr}(\lesssim 0.3 \mathrm{dex})$ on average than other PNe. We calculated $s$-process enrichments in a set of AGB models covering a range in mass from 2.5 to $6.5 M_{\odot}$ and metallicity from $0.2 Z_{\odot}$ to $Z_{\odot}$. The $2.5 M_{\odot}, Z=0.008$ model was included to show an example of a model that would produce a clear nonType I PN abundance signature, with $[\mathrm{Se}, \mathrm{Kr} / \mathrm{Fe}]>0.5$ and $\mathrm{C} / \mathrm{O} \sim 4$. The main conclusion is that the results for 3-6.5 $M_{\odot}$ are a good match to the observed abundances. The only real exception out of the HBB models is the low-metallicity $5 M_{\odot}$, $Z=0.004$ model that produced much larger enhancements in $\mathrm{Se}$ and $\mathrm{Kr}$ than observed. This suggests that Galactic Type I PNe do not descend from such low-metallicity objects.

We also compare calculated abundances for selected intermediate-mass ( $\mathrm{Ne}, \mathrm{P}, \mathrm{S}, \mathrm{Ar})$ and iron-peak ( $\mathrm{Fe}, \mathrm{Zn})$ elements to observations of post-AGB stars and PNe. We find that the elemental abundances of $\mathrm{P}, \mathrm{S}, \mathrm{Cl}, \mathrm{Ar}, \mathrm{Fe}$, and $\mathrm{Zn}$ are essentially unchanged by AGB nucleosynthesis, although there are isotopic shifts caused by neutron captures in the He-shell. These results justify use of elements such as $\mathrm{S}, \mathrm{Cl}$, and $\mathrm{Ar}$ in $\mathrm{PNe}$ as tracers of Galactic chemical evolution.

It is difficult to reach firm conclusions about the neutron source operating in massive AGB stars from Se and $\mathrm{Kr}$ abundances in Type I PNe. Certainly, it seems that at the least, the ${ }^{22} \mathrm{Ne}$ source, with efficient TDU, is required to produce enhancements in $s$-process elements in massive Type I PN progenitors. It is less clear whether a ${ }^{13} \mathrm{C}$ pocket is also required in these stars. Increases in the $[\mathrm{Kr} / \mathrm{Fe}]$ abundance ratio were observed in the models when a ${ }^{13} \mathrm{C}$ pocket was included, but not beyond the amounts observed in Type I PN spectra. 
Obtaining abundances for more $n$-capture elements, particularly $\mathrm{Rb}$ and elements beyond the first $s$-process peak, from a large data set of $\mathrm{OH} / \mathrm{IR}$ stars and $\mathrm{PNe}$ will help distinguish among the possibilities.

Finally, only the models with $\mathrm{HBB}\left(M \gtrsim 5 M_{\odot}\right.$, depending on $Z$ ) show the high $\mathrm{He} / \mathrm{H}$ and $\mathrm{N} / \mathrm{O}$ ratios that define the Type I PN class. Given that $3 M_{\odot}$ stars are more common than $6.5 M_{\odot}$ stars (according to initial mass function (IMF) and evolutionary time considerations), it may be necessary that another mixing process other than $\mathrm{HBB}$ is active in stars of the lower-mass range $\left(3-4 M_{\odot}\right)$, if these stars do in fact evolve into Type I PNe. Rapid stellar rotation in single $\sim 3 M_{\odot}$ stars may, for example, be able to account for the increased $\mathrm{He}$ and $\mathrm{N}$ abundances in the progenitor stars. The problems of convection and mixing and of other modeling uncertainties (e.g., $n$-capture cross sections) requires further study in the context of $s$-process nucleosynthesis in intermediate-mass AGB stars.

We thank R. Izzard, D. Yong, and K. Farouqi for discussions about stellar nucleosynthesis; M. Cantiello and S. Chita for discussions about stellar rotation; and R. Humble for help with the postprocessing code. A.I.K. acknowledges support from the Australian Research Council's Discovery Projects funding scheme (project number DP0664105); partial support was provided by the Joint Theory Institute funded together by Argonne National Laboratory and the University of Chicago. A.I.K. also thanks the Netherlands Organisation for Scientific Research (NWO) and the Netherlands Research School for Astronomy (NOVA) for money to visit Utrecht. M.L. is supported by the NWO through the VENI fellowship scheme. N.C.S. is supported by an appointment to the NASA Postdoctoral Program at the Goddard Space Flight Center, administered by Oak Ridge Associated Universities through a contract with NASA. H.L.D. is supported by the National Science Foundation through NSF grants AST 0406809 and 0708245.

\section{REFERENCES}

Abia, C., Busso, M., Gallino, R., Domínguez, I., Straniero, O., \& Isern, J. 2001, ApJ, 559, 1117

Abia, C., \& Isern, J. 1997, MNRAS, 289, L11

Abia, C., et al. 2002, ApJ, 579, 817

Akerman, C. J., Ellison, S. L., Pettini, M., \& Steidel, C. C. 2005, A\&A, 440, 499

Aller, L. H., \& Czyzak, S. J. 1983, ApJS, 51, 211

Aller, L. H., Keyes, C. D., Maran, S. P., Gull, T. R., Michalitsianos, A. G., \& Stecher, T. P. 1987, ApJ, 320, 159

Anders, E., \& Grevesse, N. 1989, Geochim. Cosmochim. Acta, 53, 197

Asplund, M., Grevesse, N., \& Sauval, A. J. 2005, in ASP Conf. Ser. 336, Cosmic Abundances as Records of Stellar Evolution and Nucleosynthesis, ed. T. G. Barnes, III, \& F. N. Bash (San Francisco, CA: ASP), 25

Balick, B., \& Frank, A. 2002, ARA\&A, 40, 439

Bao, Z. Y., Beer, H., Käppeler, F., Voss, F., Wisshak, K., \& Rauscher, T. 2000, Atomic Data Nucl. Data Tables, 76, 70

Bernard-Salas, J., Pottasch, S. R., Gutenkunst, S., Morris, P. W., \& Houck, J. R. 2008, ApJ, 672, 274

Boothroyd, A. I., \& Sackmann, I.-J. 1988, ApJ, 328, 653

Busso, M., Gallino, R., Lambert, D. L., Travaglio, C., \& Smith, V. V. 2001, ApJ, 557,802

Busso, M., Gallino, R., \& Wasserburg, G. J. 1999, ARA\&A, 37, 239

Calvet, N., \& Peimbert, M. 1983, RevMexAA, 5, 319

Cameron, A. G. W. 1960, AJ, 65, 485

Cayrel, R., et al. 2004, A\&A, 416, 1117

Charbonnel, C., \& Zahn, J.-P. 2007, A\&A, 467, L15

Chayer, P., Vennes, S., Dupuis, J., \& Kruk, J. W. 2005, ApJ, 630, L169

Corradi, R. L. M., \& Schwarz, H. E. 1995, A\&A, 293, 871

Cosner, K., Iben, I., Jr., \& Truran, J. W. 1980, ApJ, 238, L91

Dearborn, D. S. P., Lattanzio, J. C., \& Eggleton, P. P. 2006, ApJ, 639, 405
Deupree, R. G., \& Karakas, A. I. 2005, ApJ, 633, 418

Dinerstein, H. L. 2001, ApJ, 550, L223

Dinerstein, H. L. 2004, in Carnegie Observatories Astrophysics Series, Vol. 4: Origin and Evolution of the Elements, ed. A. McWilliam \& M. Rauch (Pasadena, CA: Carnegie Observatories), http://www.ociw.edu/ociw/ symposia/series/symposium4/proceedings.html

Dinerstein, H. L., \& Geballe, T. R. 2001, ApJ, 562, 515

Dinerstein, H. L., Geballe, T. R., Wood, J. L., \& Sterling, N. C. 2007, Bull. AAS, 39,913

Dinerstein, H. L., Richter, M. J., Lacy, J. H., \& Sellgren, K. 2003, AJ, 125, 265

Dobrinčić, M., Villaver, E., Guerrero, M. A., \& Manchado, A. 2008, AJ, 135, 2199

Dopita, M. A., \& Meatheringham, S. J. 1991, ApJ, 377, 480

Dopita, M. A., et al. 1997, ApJ, 474, 188

Dudziak, G., Péquignot, D., Zijlstra, A. A., \& Walsh, J. R. 2000, A\&A, 363, 717

Eggleton, P. P., Dearborn, D. S. P., \& Lattanzio, J. C. 2006, Science, 314, 1580

Fenner, Y., Gibson, B. K., Lee, H.-C., Karakas, A. I., Lattanzio, J. C., Chieffi, A., Limongi, M., \& Yong, D. 2003, PASA, 20, 340

Forestini, M., \& Charbonnel, C. 1997, A\&AS, 123, 241

Frost, C. A., Cannon, R. C., Lattanzio, J. C., Wood, P. R., \& Forestini, M. 1998, A\&A, 332, L17

Gallino, R., Arlandini, C., Busso, M., Lugaro, M., Travaglio, C., Straniero, O., Chieffi, A., \& Limongi, M. 1998, ApJ, 497, 388

García-Hernández, D. A., García-Lario, P., Plez, B., D’Antona, F., Manchado, A., \& Trigo-Rodríguez, J. M. 2006, Science, 314, 1751

García-Segura, G., Langer, N., Rózyczka, M., \& Franco, J. 1999, ApJ, 517, 767

Goriely, S., \& Mowlavi, N. 2000, A\&A, 362, 599

Górny, S. K., Stasińska, G., Escudero, A. V., \& Costa, R. D. D. 2004, A\&A, 427,231

Gorny, S. K., Stasińska, G., \& Tylenda, R. 1997, A\&A, 318, 256

Henry, R. B. C., Kwitter, K. B., \& Balick, B. 2004, AJ, 127, 2284

Herwig, F. 2000, A\&A, 360, 952

Herwig, F. 2004, ApJ, 605, 425

Herwig, F. 2005, ARA\&A, 43, 435

Herwig, F., Freytag, B., Hueckstaedt, R. M., \& Timmes, F. X. 2006, ApJ, 642, 1057

Herwig, F., Langer, N., \& Lugaro, M. 2003, ApJ, 593, 1056

Idiart, T. P., Maciel, W. J., \& Costa, R. D. D. 2007, A\&A, 472, 101

Kaeppeler, F., et al. 1994, ApJ, 437, 396

Kahane, C., Dufour, E., Busso, M., Gallino, R., Lugaro, M., Forestini, M., \& Straniero, O. 2000, A\&A, 357, 669

Karakas, A. I., \& Lattanzio, J. C. 2003a, PASA, 20, 393

Karakas, A. I., \& Lattanzio, J. C. 2003b, PASA, 20, 279

Karakas, A. I., \& Lattanzio, J. C. 2007, PASA, 24, 103

Karakas, A. I., Lattanzio, J. C., \& Pols, O. R. 2002, PASA, 19, 515

Karakas, A. I., Lugaro, M., \& Gallino, R. 2007, ApJ, 656, L73

Karakas, A. I., Lugaro, M., Wiescher, M., Goerres, J., \& Ugalde, C. 2006, ApJ, 643,471

Kingsburgh, R. L., \& Barlow, M. J. 1994, MNRAS, 271, 257

Lambert, D. L., Smith, V. V., Busso, M., Gallino, R., \& Straniero, O. 1995, ApJ, 450,302

Lattanzio, J., Frost, C., Cannon, R., \& Wood, P. R. 1996, Mem. Soc. Astron. Ital., 67, 729

Lattanzio, J. C. 1986, ApJ, 311, 708

Leisy, P., \& Dennefeld, M. 2006, A\&A, 456, 451

Lewis, R. S., Amari, S., \& Anders, E. 1994, Geochim. Cosmochim. Acta, 58, 471

Lodders, K. 2003, ApJ, 591, 1220

Lugaro, M., Davis, A. M., Gallino, R., Pellin, M. J., Straniero, O., \& Käppeler, F. 2003a, ApJ, 593, 486

Lugaro, M., Herwig, F., Lattanzio, J. C., Gallino, R., \& Straniero, O. 2003b, ApJ, 586, 1305

Lugaro, M., Ugalde, C., Karakas, A. I., Görres, J., Wiescher, M., Lattanzio, J. C., \& Cannon, R. C. 2004, ApJ, 615, 934

Marigo, P., Bernard-Salas, J., Pottasch, S. R., Tielens, A. G. G. M., \& Wesselius, P. R. 2003, A\&A, 409, 619

Meakin, C. A., \& Arnett, D. 2007, ApJ, 667, 448

Meléndez, J., \& Cohen, J. G. 2007, ApJ, 659, L25

Miksa, S., Deetjen, J. L., Dreizler, S., Kruk, J. W., Rauch, T., \& Werner, K. 2002, A\&A, 389, 953

Moe, M., \& De Marco, O. 2006, ApJ, 650, 916

Mucciarelli, A., Carretta, E., Origlia, L., \& Ferraro, F. R. 2008, AJ, 136, 375

Nollett, K. M., Busso, M., \& Wasserburg, G. J. 2003, ApJ, 582, 1036

Peimbert, M. 1978, in IAU Symp. 76, Planetary Nebulae, ed. Y. Terzian (Dordrecht: Kluwer), 215

Peimbert, M. 1990, Reports Prog. Phys., 53, 1559 
Pequignot, D., \& Baluteau, J.-P. 1994, A\&A, 283, 593

Péquignot, D., Walsh, J. R., Zijlstra, A. A., \& Dudziak, G. 2000, A\&A, 361, L1 Perinotto, M., Bencini, C. G., Pasquali, A., Manchado, A., Rodriguez Espinosa, J. M., \& Stanga, R. 1999, A\&A, 347, 967

Plez, B., Smith, V. V., \& Lambert, D. L. 1993, ApJ, 418, 812

Pompeia, L., et al. 2006, arXiv:astro-ph/0604009

Pottasch, S. R., \& Bernard-Salas, J. 2006, A\&A, 457, 189

Ramírez, I., Allende Prieto, C., \& Lambert, D. L. 2007, A\&A, 465, 271

Rauch, T., Werner, K., Ziegler, M., Koesterke, L., \& Kruk, J. W. 2008, in IAU Symp. 252, The Art of Modeling Stars in the 21 st Century, ed. L. Deng \& K. L. Chan (Dordrecht: Kluwer), 223

Reimers, D. 1975, in Problems in Stellar Atmospheres and Envelopes (New York: Springer), 229

Sembach, K. R., Steidel, C. C., Macke, R. J., \& Meyer, D. M. 1995, ApJ, 445, L27

Sharpee, B., Zhang, Y., Williams, R., Pellegrini, E., Cavagnolo, K., Baldwin, J. A., Phillips, M., \& Liu, X.-W. 2007, ApJ, 659, 1265

Simmerer, J., Sneden, C., Cowan, J. J., Collier, J., Woolf, V. M., \& Lawler, J. E. 2004, ApJ, 617, 1091

Smith, V. V., \& Lambert, D. L. 1989, ApJ, 345, L75

Smith, V. V., \& Lambert, D. L. 1990, ApJS, 72, 387

Smith, V. V., Lambert, D. L., \& McWilliam, A. 1987, ApJ, 320, 862

Soker, N. 1997, ApJS, 112, 487

Stancliffe, R. J., Tout, C. A., \& Pols, O. R. 2004, MNRAS, 352, 984

Stanghellini, L., Guerrero, M. A., Cunha, K., Manchado, A., \& Villaver, E. 2006, ApJ, 651, 898

Stanghellini, L., Shaw, R. A., Balick, B., \& Blades, J. C. 2000, ApJ, 534, L167

Stasińska, G., Richer, M. G., \& McCall, M. L. 1998, A\&A, 336, 667

Sterling, N. C., \& Dinerstein, H. L. 2008, ApJS, 174, 158

Sterling, N. C., Dinerstein, H. L., \& Bowers, C. W. 2002, ApJ, 578, L55
Sterling, N. C., Dinerstein, H. L., Bowers, C. W., \& Redfield, S. 2005, ApJ, 625, 368

Sterling, N. C., Dinerstein, H. L., \& Kallman, T. R. 2007, ApJS, 169, 37

Straniero, O., Chieffi, A., Limongi, M., Busso, M., Gallino, R., \& Arlandini, C. 1997, ApJ, 478, 332

Straniero, O., Gallino, R., Busso, M., Chiefei, A., Raiteri, C. M., Limongi, M., \& Salaris, M. 1995, ApJ, 440, L85

Travaglio, C., Gallino, R., Arnone, E., Cowan, J., Jordan, F., \& Sneden, C. 2004, ApJ, 601, 864

Truran, J. W., \& Iben, I., Jr. 1977, ApJ, 216, 797

van Raai, M. A., Lugaro, M., Karakas, A. I., \& García-Hernández, D. A. 2008, in AIP Conf. Ser. 1001, Evolution and Nucleosynthesis in AGB Stars (New York: AIP), 146

van Winckel, H. 2003, ARA\&A, 41, 391

Vassiliadis, E., \& Wood, P. R. 1993, ApJ, 413, 641

Ventura, P., \& D'Antona, F. 2005, A\&A, 431, 279

Ventura, P., D'Antona, F., \& Mazzitelli, I. 2002, A\&A, 393, 215

Welty, D. E., Hobbs, L. M., Lauroesch, J. T., Morton, D. C., Spitzer, L., \& York, D. G. 1999, ApJS, 124, 465

Werner, K., \& Herwig, F. 2006, PASP, 118, 183

Wood, J. L., Dinerstein, H. L., Geballe, T. R., \& Sterling, N. C. 2006, BAAS, 38, 1113

Wood, P. R., Bessell, M. S., \& Fox, M. W. 1983, ApJ, 272, 99

York, D. G., \& Jura, M. 1982, ApJ, 254, 88

Zhang, Y., \& Liu, X.-W. 2005, ApJ, 631, L61

Zijlstra, A. A., Gesicki, K., Walsh, J. R., Péquignot, D., van Hoof, P. A. M., \& Minniti, D. 2006, MNRAS, 369, 875

Zinner, E. 2008, PASA, 25, 7

Zinner, E., Nittler, L. R., Gallino, R., Karakas, A. I., Lugaro, M., Straniero, O., \& Lattanzio, J. C. 2006, ApJ, 650, 350 